\title{
BRST analysis of the supersymmetric higher spin field models
}

\author{
I.L. Buchbinder ${ }^{a, b}$ and K. Koutrolikos ${ }^{a, b, c, d}$ \\ ${ }^{a}$ Department of Theoretical Physics, Tomsk State Pedagogical University, \\ Tomsk 634041, Russia \\ ${ }^{b}$ National Research Tomsk State University, \\ Tomsk 634050, Russia \\ ${ }^{c}$ Physics Division, National Technical University of Athens, \\ 15780 Zografou Campus, Athens, Greece \\ ${ }^{d}$ Institute for Theoretical Physics \& Astrophysics, Masaryk University, \\ 61137 Brno, Czech Republic \\ E-mail: joseph@tspu.edu.ru, konstantinos.koutrolikos@gmail.com
}

ABSTRACT: We develop the BRST approach for all massless integer and half-integer higher spins in 4D Minkowski space, using the two component spinor notation and develop the Lagrangian formulation for supersymmetric higher spin models. It is shown that the problem of second class constraints disappears and the BRST procedure becomes much more simple than in tensorial notation. Furthermore, we demonstrate that the BRST procedure automatically provides extra auxiliary components that belong in the set of supersymmetry auxiliary components. Finally, we demonstrate how supersymmetry transformations transformations are realized in such an approach. As a result, we conclude that the BRST approach to higher spin supersymmetric theories allows to derive both the Lagrangian and the supersymmetry transformations. Although most part of the work is devoted to massless component supersymmetric models, we also discuss generalization for massive component supersymmetric models and for superfield models.

KEYWORDS: Higher Spin Symmetry, Supersymmetric Effective Theories, BRST Symmetry ARXiv EPrint: 1510.06569 


\section{Contents}

1 Introduction $\quad 1$

2 Massless irreducible representations of the Poincaré group 3

2.1 Half-integer spin 3

2.2 Integer spin 4

3 Algebra of operators and BRST charge 5

4 BRST description for half-integer spins $\quad 9$

$\begin{array}{lll}5 & \text { BRST description of integer spin } & 11\end{array}$

6 Supersymmetric invariant theory $\quad 13$

$\begin{array}{lll}6.1 & \text { On-shell formulation } & 13\end{array}$

$\begin{array}{ll}6.2 \text { Off-shell formulation } & 15\end{array}$

$\begin{array}{lll}7 & \text { Generalization for massive higher spin supersymmetric theories } & 17\end{array}$

$\begin{array}{llr}8 & \text { Problem of superfield BRST construction } & 18\end{array}$

$\begin{array}{llr}9 & \text { Summary } & 19\end{array}$

$\begin{array}{ll}\text { A Two-component spinor notation } & 20\end{array}$

\section{Introduction}

Supersymmetry is still a very active and promising research area in modern theoretical physics with many applications in quantum field theory, mathematical physics, string theory, phenomenology of elementary particles and cosmology (see e.g. the books [1-9] and the references therein). Another actively developing area of modern theoretical physics is higher spin field theory (see e.g. the reviews $[10-17]$ and the references therein ${ }^{1}$ ). In this paper we begin a systematic formulation of a generic approach to construct the Lagrangian for supersymmetric higher spin theories. It is clear that constructing supersymmetric higher spin models, one has to face problems from the side of supersymmetry and from the side of higher spins. In particular, we can expect that off-shell Lagrangian formulation of the supersymmetric higher spin models will require finding the auxiliary fields both to closure a supersymmetry algebra off shell and for consistent higher spin field Lagrangian description.

\footnotetext{
${ }^{1} \mathrm{~A}$ brief review of the Lagrangian formulation for $4 \mathrm{D}$ massless higher spins is given in [5] as well.
} 
Four-dimensional $\mathcal{N}=1$ supersymmetric massless higher spin field models have been proposed in [18]. These models included the necessary sets of auxiliary fields to provide the Lagrangian formulation of the higher spin theory but they were on-shell theories from supersymmetric point of view. It is generally accepted that the off-shell supersymmetric models can be realized in the framework of the superfield approach where the set of the auxiliary fields relevant to off-shell supersymmetry is part of the appropriate superfield. Massless superfield models in 4D Minkowski space have been constructed in [19, 20] and in $A d S_{4}$ have been found in [21] (see a brief review in [5]). This approach was further developed in [22, 23] and some aspect were later studied in [24].

Lagrangian construction for the massive on-shell $4 \mathrm{D}, \mathcal{N}=1$ higher superspin multiplets in flat space was realized in [25] and for the massive 3D supermultiplets in flat space in [26]. ${ }^{2}$ Some examples of the $4 \mathrm{D}, \mathcal{N}=1$ superfield dynamical models in flat space-time have been constructed in [27-30]. General superfield Lagrangian formulation for arbitrary massive higher spin supermultiplets is undeveloped until now.

The generic approach to develop the Lagrangian formulation for higher spin field theories is realized in the framework of the BRST-BFV construction [31-34] (see also the reviews $[35,36]$ ). BRST construction allows us to derive the Lagrangians for bosonic and fermionic, massless and massive, free and interacting higher spin fields in the flat and AdS spaces of various dimensions (see e.g. [37-58] and the references therein). In this paper we will focus on the BRST construction for deriving the Lagrangian formulation of the 4D massless higher $\operatorname{spin} \mathcal{N}=1$ supersymmetric field models.

The first step of the higher spin BRST approach, is to convert all constraints required for the definition of an irreducible representations of the Poincare or AdS group, into operators acting on a Fock space. These operators should form a first class algebra in terms of commutators, therefore one can treat them as the generators of some still unknown gauge theory. The second step is the construction of the BRST charge on the base of the above constraints. The third step is deriving the equations of motion as the annihilation of the physical state by the BRST charge and finding the corresponding free Lagrangian. Interaction in such a framework means a deformation of the BRST charge. However, in many cases the above approach faces the annoying feature of second class constraints, usually related with the trace and $\gamma$-trace constraints of higher spins. The typical method of resolving this issue is to expand the Fock space by introducing extra ghost oscillators which allow the conversion of the second class constraints to first class and preserve the algebra at the same time (see e.g. $[59,60]$ for conversion procedure in general gauge theories). In this work, we demonstrate an alternate method to avoid the second class constraints in the higher spin field theories by using fields with two-component spinorial indices instead of vector indices. The advantage of this description is that the definition of the irreducible representations does not lead to second class constraints at all. Therefore, in this case the BRST approach to free, massless, integer and half-integer higher spins becomes very simple and straight forward.

\footnotetext{
${ }^{2}$ In this paper the Lagrangian formulation of 3D massless supermultiplets was considered as well.
} 
The motivation for the usage of the spinorial index notations comes from the desire to apply the BRST approach to $4 \mathrm{D} \mathcal{N}=1$ superspace and describe supersymmetric higher spin theories. The natural space were these representation live is the tensor product of left and right representation of $s l(2, R)$. We will see that the BRST approach is powerful enough tool for deriving the Lagrangian description that not only provides the appropriate compensating fields for the description of higher spin, but also naturally gives rise to part of the auxiliary states required for by supersymmetry.

Moreover, we demonstrate how supersymmetry transformations emerge in the Fock space states and we derive the exact on-shell supersymmetry transformations laws for the components of massless integer and half-integer superspin irreducible representations.

The paper is organized as follows. In section 2 we briefly review the constraints required for the description of the irreducible representations, the Lagrangian formulation and equations of motion for both integer and half-integer, massless spins. In section 3 we present the algebra of all operators involved and construct the most general BRST charge $Q$. In sections 4 and 5 we take limits of $Q$ in order to define $Q_{F}$ and $Q_{B}$, the BRST charges that describe the half-integer and integer irreducible systems. In section 6 we present, the higher spin supersymmetric Lagrangian and the supersymmetry transformations between the bosonic and fermionic theory. In sections 7 and 8 we discuss generalizations for the massive case and superspace.

\section{Massless irreducible representations of the Poincaré group}

\section{$2.1 \quad$ Half-integer spin}

The irreducible representations of the Poincaré group for massless, half-integer spin $(s+1 / 2)$ are defined by the following list of constraints: ${ }^{3}$

$$
\partial^{\beta \dot{\beta}} \psi_{\beta \alpha(s) \dot{\beta} \dot{\alpha}(s-1)}=0, \quad i \partial_{(\dot{\beta}}^{\beta} \psi_{\beta \alpha(s) \dot{\alpha}(s))}=0, \quad \square \psi_{\alpha(s+1) \dot{\alpha}(s)}=0
$$

In order to generate these constraints from the variation of a Lagrangian, we have to introduce two more compensators $\bar{\psi}_{\alpha(s-1) \dot{\alpha}(s)}$ and $\psi_{\alpha(s-1) \dot{\alpha}(s-2)}$ which also make the Lagrangian to have a gauge symmetry. This Lagrangian (up to an overall sign) has the following form

$$
\begin{aligned}
\mathcal{L}_{F}= & i \bar{\psi}^{\alpha(s) \dot{\alpha}(s+1)} \partial^{\alpha_{s+1}} \dot{\alpha}_{s+1} \psi_{\alpha(s+1) \dot{\alpha}(s)} \\
& +i\left[\frac{s}{s+1}\right] \psi^{\alpha(s+1) \dot{\alpha}(s)} \partial_{\alpha_{s+1} \dot{\alpha}_{s}} \psi_{\alpha(s) \dot{\alpha}(s-1)}+c . c . \\
& -i\left[\frac{2 s+1}{(s+1)^{2}}\right] \bar{\psi}^{\alpha(s-1) \dot{\alpha}(s)} \partial^{\alpha_{s}} \dot{\alpha}_{s} \psi_{\alpha(s) \dot{\alpha}(s-1)} \\
& +i \psi^{\alpha(s) \dot{\alpha}(s-1)} \partial_{\alpha_{s} \dot{\alpha}_{s-1}} \psi_{\alpha(s-1) \dot{\alpha}(s-2)}+c . c . \\
& -i \bar{\psi}^{\alpha(s-2) \dot{\alpha}(s-1)} \partial^{\alpha_{s-1}} \dot{\alpha}_{s-1} \psi_{\alpha(s-1) \dot{\alpha}(s-2)}
\end{aligned}
$$

\footnotetext{
${ }^{3}$ The notation $\phi_{\alpha(k) \dot{\alpha}(l)}$ means that the field $\phi$ has $k$ undotted indices $\alpha_{1} \alpha_{2} \ldots \alpha_{k}$ that are symmetrized and similarly for $l$ dotted indices.
} 
and is invariant under the transformations.

$$
\begin{aligned}
\delta_{G} \psi_{\alpha(s+1) \dot{\alpha}(s)} & =\frac{1}{s !(s+1) !} \partial_{\left(\alpha _ { s + 1 } \left(\dot{\alpha}_{s}\right.\right.} \lambda_{\alpha(s)) \dot{\alpha}(s-1))}, \\
\delta_{G} \bar{\psi}_{\alpha(s-1) \dot{\alpha}(s)} & =\frac{1}{s !} \partial^{\alpha_{s}} \dot{\alpha}_{s} \lambda_{\alpha(s) \dot{\alpha}(s-1))}, \\
\delta_{G} \psi_{\alpha(s-1) \dot{\alpha}(s-2)} & =\frac{s-1}{s} \partial^{\alpha_{s} \dot{\alpha}_{s-1}} \lambda_{\alpha(s) \dot{\alpha}(s-1)} .
\end{aligned}
$$

where $\lambda_{\alpha(s) \dot{\alpha}(s-1)}$ are the unconstrained gauge parameters. The equations of motion corresponding to the Lagrangian are

$$
\begin{aligned}
& i \partial^{\alpha_{s+1}}{ }_{\left(\dot{\alpha}_{s+1}\right.} \psi_{\alpha(s+1) \dot{\alpha}(s))}-i \frac{s}{(s+1) s !} \partial_{\left(\alpha _ { s } \left(\dot{\alpha}_{s+1}\right.\right.} \bar{\psi}_{\alpha(s-1)) \dot{\alpha}(s))}=0, \\
& i \frac{2 s+1}{s !} \partial_{\left(\alpha_{s}\right.}{ }^{\dot{\alpha}_{s}} \bar{\psi}_{\alpha(s-1) \dot{\alpha}(s)}+i s(s+1) \partial^{\dot{\alpha}_{s+1} \dot{\alpha}_{s}} \psi_{\alpha(s+1) \dot{\alpha}(s)} \\
& -i \frac{(s+1)^{2}}{s !(s-1) !} \partial_{\left(\alpha _ { s } \left(\dot{\alpha}_{s-1}\right.\right.} \psi_{\alpha(s-1)) \dot{\alpha}(s-2))}=0, \\
& \frac{i}{(s-1) !} \partial^{\alpha_{s-1}} \dot{\alpha}_{s-1} \psi_{\alpha(s-1) \dot{\alpha}(s-2))}-i \partial^{\alpha_{s-1} \dot{\alpha}_{s}} \bar{\psi}_{\alpha(s-1) \dot{\alpha}(s)}=0 \text {. }
\end{aligned}
$$

It is easy to verify that the constraints (2.1) can be derived from (2.4) once we use the gauge freedom (2.3) to gauge away $\bar{\psi}_{\alpha(s-1) \dot{\alpha}(s)}$ and $\psi_{\alpha(s-1) \dot{\alpha}(s-2)}$.

\subsection{Integer spin}

The constraints for the description of a massless, integer spin $(s)$ are

$$
\partial^{\beta \dot{\beta}} h_{\beta \alpha(s-1) \dot{\beta} \dot{\alpha}(s-1)}=0, \quad \square h_{\alpha(s) \dot{\alpha}(s)}=0
$$

The corresponding Lagrangian includes one real compensator $h_{\alpha(s-2) \dot{\alpha}(s-2)}$ and has the form

$$
\begin{aligned}
\mathcal{L}_{B}= & h^{\alpha(s) \dot{\alpha}(s)} \square h_{\alpha(s) \dot{\alpha}(s)} \\
& -\frac{s}{2} h^{\alpha(s) \dot{\alpha}(s)} \partial_{\alpha_{s} \dot{\alpha}_{s}} \partial^{\gamma \dot{\gamma}} h_{\gamma \alpha(s-1) \dot{\gamma} \dot{\alpha}(s-1)} \\
& +s(s-1) h^{\alpha(s) \dot{\alpha}(s)} \partial_{\alpha_{s} \dot{\alpha}_{s}} \partial_{\alpha_{s-1} \dot{\alpha}_{s-1}} h_{\alpha(s-2) \dot{\alpha}(s-2)} \\
& -s(2 s-1) h^{\alpha(s-2) \dot{\alpha}(s-2)} \square h_{\alpha(s-2) \dot{\alpha}(s-2)} \\
& -\left[\frac{s(s-2)^{2}}{2}\right] h^{\alpha(s-2) \dot{\alpha}(s-2)} \partial_{\alpha_{s-2} \dot{\alpha}_{s-2}} \partial^{\gamma \dot{\gamma}} h_{\gamma \alpha(s-3) \dot{\gamma} \dot{\alpha}(s-3)} .
\end{aligned}
$$

It is invariant under the following gauge transformations

$$
\begin{aligned}
\delta_{G} h_{\alpha(s) \dot{\alpha}(s)} & =\frac{1}{s ! s !} \partial_{\left(\alpha _ { s } \left(\dot{\alpha}_{s}\right.\right.} \zeta_{\alpha(s-1)) \dot{\alpha}(s-1))}, \\
\delta_{G} h_{\alpha(s-2) \dot{\alpha}(s-2)} & =\frac{s-1}{s^{2}} \partial^{\alpha_{s-1} \dot{\alpha}_{s-1}} \zeta_{\alpha(s-1) \dot{\alpha}(s-1)} .
\end{aligned}
$$


where $\zeta_{\alpha(s-1) \dot{\alpha}(s-1)}$ are the unconstrained gauge parameters. The equations of motion corresponding to the Lagrangian are

$$
\begin{aligned}
\square & h_{\alpha(s) \dot{\alpha}(s)}-\frac{s}{2 s !^{2}} \partial_{\left(\alpha _ { s } \left(\dot{\alpha}_{s}\right.\right.} \partial^{\gamma \dot{\gamma}} h_{\gamma \alpha(s-1)) \dot{\gamma} \dot{\alpha}(s-1))} \\
+\frac{s(s-1)}{2 s !^{2}} \partial_{\left(\alpha _ { s } \left(\dot{\alpha}_{s}\right.\right.} \partial_{\alpha_{s-1} \dot{\alpha}_{s-1}} h_{\alpha(s-2)) \dot{\alpha}(s-2))} & =0, \\
\square h_{\alpha(s-2) \dot{\alpha}(s-2)}+\frac{(s-2)^{2}}{2(2 s-1)(s-2) !^{2}} \partial_{\left(\alpha _ { s - 2 } \left(\dot{\alpha}_{s-2}\right.\right.} \partial^{\gamma \dot{\gamma}} h_{\gamma \alpha(s-3)) \dot{\gamma} \dot{\alpha}(s-3))} & \\
-\frac{(s-1)}{2(2 s-1)} \partial^{\beta \dot{\beta}} \partial^{\gamma \dot{\gamma}} h_{\beta \gamma \alpha(s-2) \dot{\beta} \dot{\gamma} \dot{\alpha}(s-2)} & =0 .
\end{aligned}
$$

Using the symmetry freedom (2.7) and the above equations of motion we can generate the constraints (2.5).

\section{Algebra of operators and BRST charge}

First of all we construct the Fock space by introducing two commuting pairs of creation and annihilation operators, one for the left space (undotted indices) and one for the right space (dotted indices).

$$
\begin{aligned}
& {\left[a_{\alpha}, a_{\beta}\right]=0, \quad\left[a_{\alpha}, a^{\dagger \beta}\right]=\delta_{\alpha}{ }^{\beta}, \quad\left[a^{\dagger \alpha}, a^{\dagger \beta}\right]=0,} \\
& {\left[\bar{a}_{\dot{\alpha}}, \bar{a}_{\dot{\beta}}\right]=0, \quad\left[\bar{a}_{\dot{\alpha}}, \bar{a}^{\dagger \dot{\beta}}\right]=\delta_{\dot{\alpha}}^{\dot{\beta}}, \quad\left[\bar{a}^{\dagger \dot{\alpha}}, \bar{a}^{\dagger \dot{\beta}}\right]=0,} \\
& {\left[a_{\alpha}, \bar{a}_{\dot{\beta}}\right]=0, \quad\left[a_{\alpha}, \bar{a}^{\dagger \dot{\beta}}\right]=0, \quad\left[a^{\dagger \alpha}, \bar{a}^{\dagger \dot{\beta}}\right]=0, \quad\left[a^{\dagger \alpha}, \bar{a}_{\dot{\beta}}\right]=0 \text {. }}
\end{aligned}
$$

For each one of them, we can define a vacuum state:

$$
a_{\alpha}|0\rangle=0, \bar{a}_{\dot{\alpha}}|\overline{0}\rangle=0
$$

hence, the general vector states are of the form

$$
|\Phi\rangle=\sum_{k=0}^{\infty} \sum_{l=0}^{\infty} \Phi_{\alpha(k) \dot{\alpha}(l)} a^{\dagger \alpha(k)} \bar{a}^{\dagger \dot{\alpha}(l)}|0, \overline{0}\rangle
$$

and due to the commuting properties of $a^{\dagger \alpha_{\mathrm{S}}}$ and $\bar{a}^{\dagger \dot{\alpha}_{\mathrm{S}}}$ the field $\Phi_{\alpha(k) \dot{\alpha}(l)}$, which is defined as the coefficient in the expansion of the state vector, will have the correct index symmetries for the description of higher spins (see appendix A).

The set of operators that act upon the general state vectors includes the two number operators (one for left and one for right space)

$$
N=a^{\dagger \alpha} a_{\alpha}, \bar{N}=\bar{a}^{\dagger \dot{\alpha}} \bar{a}_{\dot{\alpha}}
$$

the derivative operator (there are four combinations)

$$
\begin{aligned}
L_{1} & =\partial^{\alpha \dot{\alpha}} a_{\alpha} \bar{a}_{\dot{\alpha}}, \quad L_{-1}=-\partial_{\alpha \dot{\alpha}} a^{\dagger \alpha} \bar{a}^{\dagger \dot{\alpha}}, \\
T_{0} & =i \partial^{\alpha}{ }_{\dot{\alpha}} a_{\alpha} \bar{a}^{\dagger \dot{\alpha}}, \quad T_{0}^{\prime}=i \partial_{\alpha}{ }^{\dot{\alpha}} a^{\dagger \alpha} \bar{a}_{\dot{\alpha}}
\end{aligned}
$$


and of course, the $L_{0}=\square$ operator. The coefficients and sings in the definitions (3.5) have been chosen so under complex conjugation we get $\left[T_{0}\right]^{*}=T_{0}^{\prime},\left[L_{1}\right]^{*}=L_{1},\left[L_{-1}\right]^{*}=L_{-1}$ and under Hermitian conjugation we get $\left[T_{0}\right]^{\dagger}=T_{0},\left[T_{0}^{\prime}\right]^{\dagger}=T_{0}^{\prime},\left[L_{1}\right]^{\dagger}=L_{-1}$. The algebra of these operators is

$$
\begin{aligned}
{\left[L_{0}, L_{1}\right] } & =\left[L_{0}, L_{-1}\right]=\left[L_{0}, T_{0}\right]=\left[L_{0}, T_{0}^{\prime}\right]=\left[L_{0}, N\right]=\left[L_{0}, \bar{N}\right]=0, \\
{\left[T_{0}, L_{1}\right] } & =\left[T_{0}, L_{-1}\right]=\left[T_{0}, L_{1}\right]=\left[T_{0}^{\prime}, L_{1}\right]=\left[T_{0}^{\prime}, L_{-1}\right]=0, \\
{\left[L_{1}, L_{-1}\right] } & =-(N+\bar{N}+2) L_{0}, \quad\left[T_{0}, T_{0}^{\prime}\right]=(\bar{N}-N) L_{0}, \\
T_{0}^{\prime} T_{0} & =L_{-1} L_{1}+N(\bar{N}+1) L_{0}, T_{0} T_{0}^{\prime}=L_{-1} L_{1}+(N+1) \bar{N} L_{0} \\
{\left[L_{1}, N\right] } & =L_{1}, \quad\left[L_{-1}, N\right]=-L_{-1}, \quad\left[T_{0}, N\right]=T_{0}, \quad\left[T_{0}^{\prime}, N\right]=-T_{0}^{\prime}, \\
{\left[L_{1}, \bar{N}\right] } & =L_{1}, \quad\left[L_{-1}, \bar{N}\right]=-L_{-1}, \quad\left[T_{0}, \bar{N}\right]=-T_{0}, \quad\left[T_{0}^{\prime}, \bar{N}\right]=T_{0}^{\prime} .
\end{aligned}
$$

Using the above algebra it is possible to construct a nilpotent operator $Q\left(Q^{2}=0\right) .{ }^{4}$ For the case of massless theories (there are no dimension-full parameters) $Q$ must be of the form

$$
Q=Q^{(0)}+Q^{(1)}+Q^{(2)}
$$

where $Q^{(n)}$ includes terms with exactly $n$ derivatives. ${ }^{5}$ Therefore, $Q^{(0)}$ will be a function of $N$ and $\bar{N}$

$$
Q^{(0)}=f(N, \bar{N})
$$

$Q^{(1)}$ will be a linear combination of $L_{1}, L_{-1}, T_{0}, T_{0}^{\prime}$

$$
Q^{(1)}=\eta T_{0}+\rho T_{0}^{\prime}+\sigma L_{1}+\zeta L_{-1}
$$

and $Q^{(2)}$ will include $L_{0}$ plus all possible pair products of the one derivative operators

$$
Q^{(2)}=\xi L_{0}+\phi_{1} L_{-1} L_{1}+\phi_{2} L_{1} T_{0}+\ldots
$$

Furthermore due to nilpotency they must be such that

$$
\begin{aligned}
& {\left[Q^{(0)}\right]^{2},\left\{Q^{(0)}, Q^{(1)}\right\}=0, \quad\left\{Q^{(0)}, Q^{(2)}\right\}+\left[Q^{(1)}\right]^{2}=0,} \\
& \left\{Q^{(1)}, Q^{(2)}\right\}=0, \quad\left[Q^{(2)}\right]^{2}=0 .
\end{aligned}
$$

It is straight forward to prove that (3.11) are satisfied if

$$
\begin{aligned}
\phi_{1} & =\phi_{2}=\cdots=0, \\
\eta^{2} & =\rho^{2}=\sigma^{2}=\zeta^{2}=\xi^{2}=P_{\xi}^{2}=0, \\
\{\eta, \rho\} & =\{\sigma, \zeta\}=\{\eta, \sigma\}=\{\rho, \sigma\}=\{\eta, \zeta\}=0, \\
\{\xi, \eta\} & =\{\xi, \rho\}=\{\xi, \sigma\}=\{\xi, \zeta\}=\{\rho, \zeta\}=0, \\
\left\{P_{\xi}, \eta\right\} & =\left\{P_{\xi}, \rho\right\}=\left\{P_{\xi}, \sigma\right\}=\left\{P_{\xi}, \zeta\right\}=0, \quad\left\{\xi, P_{\xi}\right\}=1, \\
f(N, \bar{N}) & =[\eta \rho(N-\bar{N})+\sigma \zeta(N+\bar{N}+2)] P_{\xi} .
\end{aligned}
$$

\footnotetext{
${ }^{4}$ In principle nilpotency means that there is a positive number $k$ such that $Q^{k}=0$. For this case $k$ will be 2 because of the $g \times g \rightarrow g$ structure of the algebra, but in principle for more complicated cases we may have to allow for higher values.

${ }^{5}$ For a more abstract discussion $n$ does not have to stop at 2, but since we want to generate equations with up to two derivatives, it is obvious that $n \leq 2$.
} 
We conclude that

1. Based on (3.12) the objects $\eta, \rho, \sigma, \zeta, \xi$ must be interpreted as anti-commuting oscillators that expand our Fock space. These are the usual BRST ghosts and we assign them a ghost number +1 .

2. For each one of them, there is a conjugate oscillator $P_{\eta}, P_{\rho}, P_{\sigma}, P_{\zeta}, P_{\xi}$ which has a ghost number of -1 .

3. We can define a vacuum state $\left|0_{g h}\right\rangle$ for the ghost sector, as the state that is being annihilated by a subset of the above oscillators. Different choices for the vacuum state (or equivalently different choices of oscillators that annihilate the vacuum) will lead to the description of different systems.

4. The BRST charge $Q$ for algebra (3.6) is:

$$
Q=\eta T_{0}+\rho T_{0}^{\prime}+\sigma L_{1}+\zeta L_{-1}+\xi L_{0}+[\eta \rho(N-\bar{N})+\sigma \zeta(N+\bar{N}+2)] P_{\xi}
$$

5. By choosing $\sigma$ to be the hermitian conjugate of $\zeta$ and $\eta, \rho, \xi$ to be self hermitian then $Q$ becomes hermitian.

For both integer and half-integer spins $T_{0}^{\prime}, L_{-1}$ are not constraints, therefore a reasonable choice for the vacuum state is

$$
P_{\eta}\left|0_{g h}\right\rangle=\rho\left|0_{g h}\right\rangle=P_{\sigma}\left|0_{g h}\right\rangle=\zeta\left|0_{g h}\right\rangle=P_{\xi}\left|0_{g h}\right\rangle=0 .
$$

With this choice, the most general state $|\Phi\rangle$ allowed, is of the form

$$
|\Phi\rangle=\sum_{\substack{k_{1}, k_{2}, k_{3} \\ l_{1}, l_{2}}} \eta^{k_{1}} \sigma^{k_{2}} \xi^{k_{3}} P_{\zeta}^{l_{1}} P_{\xi}^{l_{2}}\left|\Phi_{k_{1}, k_{2}, K_{3}, l_{1}, l_{2}}\right\rangle
$$

where the $k$ s and the $l \mathrm{~s}$ can take two values, zero or one and we sum over them. The ghost number value for the general term in the sum is (we choose the vacuum to have zero ghost number)

$$
g h(|\Phi\rangle)=k_{1}+k_{2}+k_{3}-l_{1}-l_{2}
$$

Therefore the zero ghost state, which will play the role of the physical state $(Q|\Psi\rangle=0)$ is

$$
\begin{aligned}
|\Psi\rangle= & |S\rangle+\eta P_{\zeta}|A\rangle+\sigma P_{\zeta}|B\rangle+\xi P_{\zeta}|\Gamma\rangle \\
& +\eta P_{\rho}|U\rangle+\sigma P_{\rho}|V\rangle+\xi P_{\rho}|\Delta\rangle \\
& +\eta \sigma P_{\zeta} P_{\rho}|W\rangle+\eta \xi P_{\zeta} P_{\rho}|Z\rangle+\sigma \xi P_{\zeta} P_{\rho}|H\rangle
\end{aligned}
$$

the -1 ghost state, which will play the role of the gauge parameter for the transformation of the physical state $\left(\delta_{G}|\Psi\rangle=Q|\Lambda\rangle\right)$ is

$$
r l|\Lambda\rangle=P_{\zeta}|\lambda\rangle+P_{\rho}|\kappa\rangle+\eta P_{\zeta} P_{\rho}|\pi\rangle+\sigma P_{\zeta} P_{\rho}|\tau\rangle+\xi P_{\zeta} P_{\rho}|\Upsilon\rangle
$$


and the -2 ghost state which will play the role of a gauge parameter for a second level gauge transformation $\left(\delta_{G}|\Lambda\rangle=Q|\Xi\rangle\right)$ is

$$
r l|\Xi\rangle=P_{\zeta} P_{\rho}|\omega\rangle
$$

Now we can find the equations of motion for the components as defined above:

$$
\begin{aligned}
T_{0}|S\rangle-L_{-1}|A\rangle-T_{0}^{\prime}|U\rangle+(N-\bar{N})|\Delta\rangle & =0, \\
T_{0}^{\prime}|V\rangle-L_{1}|S\rangle+L_{-1}|B\rangle+(N+\bar{N}+2)|\Gamma\rangle & =0, \\
T_{0}|B\rangle-L_{1}|A\rangle-T_{0}^{\prime}|W\rangle-(N-\bar{N})|H\rangle & =0, \\
T_{0}|V\rangle-L_{1}|U\rangle+L_{-1}|W\rangle-(N+\bar{N}+2)|Z\rangle & =0, \\
L_{0}|S\rangle-L_{-1}|\Gamma\rangle-T_{0}^{\prime}|\Delta\rangle & =0, \\
L_{0}|A\rangle-T_{0}|\Gamma\rangle+T_{0}^{\prime}|Z\rangle & =0, \\
L_{0}|B\rangle-L_{1}|\Gamma\rangle+T_{0}^{\prime}|H\rangle & =0, \\
L_{0}|U\rangle-L_{-1}|Z\rangle-T_{0}|\Delta\rangle & =0, \\
L_{0}|V\rangle-L_{1}|\Delta\rangle-L_{-1}|H\rangle & =0, \\
L_{0}|W\rangle-L_{1}|Z\rangle+T_{0}|H\rangle & =0,
\end{aligned}
$$

and they are invariant under the following transformations

$$
\begin{array}{rlrl}
\delta_{G}|S\rangle & =L_{-1}|\lambda\rangle+T_{0}^{\prime}|\kappa\rangle, & \delta_{G}|V\rangle & =L_{1}|\kappa\rangle-L_{-1}|\tau\rangle+(N+\bar{N}+2)|\Upsilon\rangle \\
\delta_{G}|A\rangle & =T_{0}|\lambda\rangle+T_{0}^{\prime}|\pi\rangle-(N-\bar{N})|\Upsilon\rangle, & \delta_{G}|\Delta\rangle & =L_{0}|\kappa\rangle-L_{-1}|\Upsilon\rangle \\
\delta_{G}|B\rangle & =L_{1}|\lambda\rangle+T_{0}^{\prime}|\tau\rangle, & \delta_{G}|W\rangle & =T_{0}|\tau\rangle-L_{1}|\pi\rangle \\
\delta_{G}|\Gamma\rangle & =L_{0}|\lambda\rangle+T_{0}^{\prime}|\Upsilon\rangle, & \delta_{G}|Z\rangle & =-L_{0}|\pi\rangle+T_{0}|\Upsilon\rangle \\
\delta_{G}|U\rangle & =T_{0}|\kappa\rangle-L_{-1}|\pi\rangle, & \delta_{G}|H\rangle & =-L_{0}|\tau\rangle+L_{1}|\Upsilon\rangle \\
\delta_{G}|\lambda\rangle & =-T_{0}^{\prime}|\omega\rangle, & & \\
\delta_{G}|\kappa\rangle & =L_{-1}|\omega\rangle, & \delta_{G}|\Upsilon\rangle & =L_{1}|\omega\rangle \\
\delta_{G}|\pi\rangle & =T_{0}|\omega\rangle
\end{array}
$$

It is obvious, that the above construction does not describe an irreducible representation but gives the most general BRST charge that can be constructed out of the specific set of operators. Therefore, the BRST charges responsible for the irreducible representations must be able to be derived out of it. We observe that the algebra (3.6) has three subalgebras

1. $\left\{T_{0}, L_{1}, L_{-1}, L_{0}, N, \bar{N}\right\}$

2. $\left\{T_{0}^{\prime}, L_{1}, L_{-1}, L_{0}, N, \bar{N}\right\}$

3. $\left\{L_{1}, L_{-1}, L_{0}, N, \bar{N}\right\}$

The first one includes the constraints required for the description of massless half-integer spins. The second set is related to the first one via a complex conjugation. Therefore, it 
describes the same representation as seen from the complex conjugated viewpoint. Finally, the third subalgebra includes the constraints for the description of massless integer spins. In this case all the operators involved are real therefore we can have a real representation.

\section{BRST description for half-integer spins}

To construction the BRST charge generated by the subalgebra of $\left\{T_{0}, L_{1}, L_{-1}, L_{0}, N, \bar{N}\right\}$ all we have to do is start with the general BRST charge (3.13) and freeze out the $\rho, P_{\rho}$ pair of oscillators from the Fock space. We can do that by ignoring all terms in $Q$ that include $\rho$ (naive $\rho \rightarrow 0$ limit). Hence, the BRST charge for the description of massless, half-integer spin, fermions is

$$
Q_{F}=\eta T_{0}+\sigma L_{1}+\zeta L_{-1}+\xi L_{0}+\sigma \zeta(N+\bar{N}+2) P_{\xi} .
$$

By taking the same limit in equations (3.17), (3.18), (3.19), (3.20) we find the physical state (zero ghost state) to be

$$
r l\left|\Psi_{F}\right\rangle=\left|S_{F}\right\rangle+\eta P_{\zeta}\left|A_{F}\right\rangle+\sigma P_{\zeta}\left|B_{F}\right\rangle+\xi P_{\zeta}\left|\Gamma_{F}\right\rangle
$$

the gauge parameter state (-1 ghost state)

$$
r l\left|\Lambda_{F}\right\rangle=P_{\zeta}\left|\lambda_{F}\right\rangle
$$

and there is no second level gauge parameter (-2 ghost state). The equations of motion are

$$
\begin{aligned}
T_{0}\left|S_{F}\right\rangle-L_{-1}\left|A_{F}\right\rangle & =0 \\
L_{1}\left|S_{F}\right\rangle-L_{-1}\left|B_{F}\right\rangle+(N+\bar{N}+2)\left|\Gamma_{F}\right\rangle & =0 \\
T_{0}\left|B_{F}\right\rangle-L_{1}\left|A_{F}\right\rangle & =0 \\
L_{0}\left|S_{F}\right\rangle-L_{-1}\left|\Gamma_{F}\right\rangle & =0 \\
L_{0}\left|A_{F}\right\rangle-T_{0}\left|\Gamma_{F}\right\rangle & =0 \\
L_{0}\left|B_{F}\right\rangle-L_{1}\left|\Gamma_{F}\right\rangle & =0
\end{aligned}
$$

and they are invariant under the following transformations

$$
\begin{aligned}
\delta_{G}\left|S_{F}\right\rangle & =L_{-1}\left|\lambda_{F}\right\rangle, & \delta_{G}\left|B_{F}\right\rangle & =L_{1}\left|\lambda_{F}\right\rangle, \\
\delta_{G}\left|A_{F}\right\rangle & =T_{0}\left|\lambda_{F}\right\rangle, & \delta_{G}\left|\Gamma_{F}\right\rangle & =L_{0}\left|\lambda_{F}\right\rangle .
\end{aligned}
$$

At this point, it is obvious that this system indeed describes a massless half-integer irreducible representation, because the gauge transformations of the three components $\left|S_{F}\right\rangle,\left|A_{F}\right\rangle,\left|B_{F}\right\rangle$ match gauge transformations (2.3) of the three components required for the description of the irreducible representation and the forth component $\left|\Gamma_{F}\right\rangle$ is auxiliary since its equation of motion (4.4b) is algebraic. As a consequence the dynamics of the two systems must be the same. 
However, we can demonstrate that further. $\left|\Gamma_{F}\right\rangle$ component is auxiliary due to the fact that it has higher mass dimension, because the oscillator $\xi$ is the coefficient of the $\square$ operator in the BRST charge $Q_{F}$. Therefore, we are allowed to do a redefinition of it in terms of the other states $\left|S_{F}\right\rangle,\left|A_{F}\right\rangle,\left|B_{F}\right\rangle$. Of course, this redefinition must be compatible with the gauge transformation of $\left|\Gamma_{F}\right\rangle$. Not only that, but we should be able to use this redefinition to make $\left|\Gamma_{F}\right\rangle$ 1) vanish on-shell and 2) make it gauge invariant. With that in mind, consider the ansatz

$$
\left|\Gamma_{F}\right\rangle=g_{1} T_{0}^{\prime}\left|A_{F}\right\rangle+g_{2} L_{1}\left|S_{F}\right\rangle+g_{3} L_{-1}\left|B_{F}\right\rangle+|\beta\rangle
$$

In order the left hand side to be compatible with the right hand side and our demand for $\delta_{G}|\beta\rangle=0$, we must have

$$
g_{1}+g_{2}+g_{3}=0, g_{1} N(\bar{N}+1)-g_{2}(N+\bar{N}+2)=1 .
$$

The solution of the above gives the redefinition

$$
\begin{aligned}
\left|\Gamma_{F}\right\rangle= & \frac{g}{N+\bar{N}+2}\left\{(N+\bar{N}+2) T_{0}^{\prime}\left|A_{F}\right\rangle+N(\bar{N}+1) L_{1}\left|S_{F}\right\rangle\right. \\
& \left.-(N+1)(\bar{N}+2) L_{-1}\left|B_{F}\right\rangle\right\} \\
& -\frac{1}{N+\bar{N}+2}\left\{L_{1}\left|S_{F}\right\rangle-L_{-1}\left|B_{F}\right\rangle\right\}+|\beta\rangle, \quad \forall g
\end{aligned}
$$

and the substitution of this expression to (4.4) gives the following equations

$$
\begin{aligned}
\left|\mathcal{E}_{1}\right\rangle & =T_{0}\left|S_{F}\right\rangle-L_{-1}\left|A_{F}\right\rangle=0 \\
\left|\mathcal{E}_{2}\right\rangle & =(N+\bar{N}+2) T_{0}^{\prime}\left|A_{F}\right\rangle+N(\bar{N}+1) L_{1}\left|S_{F}\right\rangle-(N+1)(\bar{N}+2) L_{-1}\left|B_{F}\right\rangle=0 \\
\left|\mathcal{E}_{3}\right\rangle & =T_{0}\left|B_{F}\right\rangle-L_{1}\left|A_{F}\right\rangle=0 \\
\left|\mathcal{E}_{4}\right\rangle & =L_{0}\left|S_{F}\right\rangle+\frac{1}{N+\bar{N}} L_{-1} L_{1}\left|S_{F}\right\rangle-\frac{1}{N+\bar{N}} L_{-1} L_{-1}\left|B_{F}\right\rangle=0 \\
\left|\mathcal{E}_{5}\right\rangle & =\frac{2(N+\bar{N}+3)}{N+\bar{N}+4} L_{0}\left|B_{F}\right\rangle-\frac{1}{N+\bar{N}+4} L_{-1} L_{1}\left|B_{F}\right\rangle+\frac{1}{N+\bar{N}+4} L_{1} L_{1}\left|S_{F}\right\rangle=0 \\
\left|\mathcal{E}_{6}\right\rangle & =|\beta\rangle=0
\end{aligned}
$$

Equations (4.9) are exactly the equations of motion for a massless half-integer spin (2.4). Equations (4.10) are quadratic in derivatives and have the structure of integer spin equations (2.8). However, these equations are not extra constraints because they are automatically satisfied if $\left|\mathcal{E}_{1}\right\rangle,\left|\mathcal{E}_{2}\right\rangle,\left|\mathcal{E}_{3}\right\rangle$ hold. This is due to the following identities

$$
\begin{aligned}
T_{0}^{\prime}\left|\mathcal{E}_{1}\right\rangle+\frac{1}{N+\bar{N}} L_{-1}\left|\mathcal{E}_{3}\right\rangle & =N(\bar{N}+1)\left|\mathcal{E}_{4}\right\rangle, \\
T_{0}^{\prime}\left|\mathcal{E}_{2}\right\rangle+\frac{1}{N+\bar{N}+4} L_{1}\left|\mathcal{E}_{3}\right\rangle & =(N+1)(\bar{N}+2)\left|\mathcal{E}_{5}\right\rangle .
\end{aligned}
$$

Equation (4.11) is the statement that the theory has a gauge invariant auxiliary component. Exactly this type of components appear in the off-shell supersymmetric spectrum of the 
irreducible representations of the super-Poincaré group and it is very interesting that BRST is powerful enough to signal about their presence.

Equations (4.9), (4.11) and can be deduced from the Lagrangian ${ }^{6}$

$$
\begin{aligned}
\mathcal{L}= & \left\langle S_{F}\left|T_{0}\right| S_{F}\right\rangle \\
& -\left\langle S_{F}\left|L_{-1}\right| A_{F}\right\rangle-\left\langle A_{F}\left|L_{1}\right| S_{F}\right\rangle \\
& -\left\langle A_{F}\left|\frac{N+\bar{N}+2}{N(\bar{N}+1)} T_{0}^{\prime}\right| A_{F}\right\rangle \\
& +\left\langle A_{F}\left|\frac{(N+1)(\bar{N}+2)}{N(\bar{N}+1)} L_{-1}\right| B_{F}\right\rangle+\left\langle B_{F}\left|\frac{(N+3)(\bar{N}+2)}{(N+2)(\bar{N}+1)} L_{1}\right| A_{F}\right\rangle \\
& -\left\langle B_{F}\left|\frac{(N+3)(\bar{N}+2)}{(N+2)(\bar{N}+1)} T_{0}\right| B_{F}\right\rangle \\
& +\langle\rho \mid \beta\rangle+\langle\beta \mid \rho\rangle
\end{aligned}
$$

where $|\rho\rangle$ is a lagrange multiplier that is required in order to generate the algebraic equation of $|\beta\rangle$, since the mass dimensions of $|\beta\rangle$ do not allow it to appear in a quadratic way in the lagrangian. A very intriguing observation is that the above Lagrangian corresponds to the fermionic part of the superspace action that describes massless integer and half-integer superspin theories. The fields $\psi_{\alpha(s+1) \dot{\alpha}(s)}, \bar{\psi}_{\alpha(s-1) \dot{\alpha}(s)}, \psi_{\alpha(s-1) \dot{\alpha}(s-2)}$ in (2.4) correspond to the states $\left|S_{F}\right\rangle,\left|A_{F}\right\rangle,\left|B_{F}\right\rangle$ in (4.9) and the auxiliary states $|\beta\rangle$, $|\rho\rangle$ will correspond to the auxiliary field $\beta_{\alpha(s) \dot{\alpha}(s-1)}, \rho_{\alpha(s) \dot{\alpha}(s-1)}$ respectively.

\section{$5 \quad$ BRST description of integer spin}

For the massless integer spin representations, we focus at the subalgebra generated by the set $\left\{L_{1}, L_{-1}, L_{0}, N, \bar{N}\right\}$. In this case we have to freeze both $\eta$ and $\rho$ oscillators. Hence, the expression for the integer spin BRST charge, $Q_{B}$ is

$$
Q_{B}=\sigma L_{1}+\zeta L_{-1}+\xi L_{0}+\sigma \zeta(N+\bar{N}+2) P_{\xi}
$$

The physical state (zero ghost state) is

$$
\left|\Psi_{B}\right\rangle=\left|S_{B}\right\rangle+\sigma P_{\zeta}\left|B_{B}\right\rangle+\xi P_{\zeta}\left|\Gamma_{B}\right\rangle
$$

and the gauge parameter state $(-1$ ghost state) is

$$
\left|\Lambda_{B}\right\rangle=P_{\zeta}\left|\lambda_{B}\right\rangle .
$$

Immediatly we get the following equation of motion

$$
\begin{aligned}
L_{1}\left|S_{B}\right\rangle-L_{-1}\left|B_{B}\right\rangle+(N+\bar{N}+2)\left|\Gamma_{B}\right\rangle & =0, \\
L_{0}\left|S_{B}\right\rangle-L_{-1}\left|\Gamma_{B}\right\rangle & =0, \\
L_{0}\left|B_{B}\right\rangle-L_{1}\left|\Gamma_{B}\right\rangle & =0 .
\end{aligned}
$$

\footnotetext{
${ }^{6} \mathrm{Up}$ to an overall factor.
} 
which are invariant under the following gauge transformations

$$
\begin{aligned}
\delta_{G}\left|S_{B}\right\rangle & =L_{-1}\left|\lambda_{B}\right\rangle, \quad \delta_{G}\left|\Gamma_{B}\right\rangle=L_{0}\left|\lambda_{B}\right\rangle, \\
\delta_{G}\left|B_{B}\right\rangle & =L_{1}\left|\lambda_{B}\right\rangle .
\end{aligned}
$$

As in the previous case, the state $\left|\Gamma_{B}\right\rangle$ is auxiliary and therefore can be redefined in order to make it vanish on-shell and gauge invariant at the same time. Since there is no state $|A\rangle$ this time, we can get the expression for the redefinition of $\left|\Gamma_{B}\right\rangle$ by taking the $g \rightarrow$ 0 limit of (4.8)

$$
\left|\Gamma_{B}\right\rangle=-\frac{1}{N+\bar{N}+2}\left\{L_{1}\left|S_{B}\right\rangle-L_{-1}\left|B_{B}\right\rangle\right\}+|\gamma\rangle
$$

Plugging in this redefinition into (5.4) we get the equation of motion

$$
\begin{aligned}
& \left|\mathcal{Z}_{1}\right\rangle=L_{0}\left|S_{B}\right\rangle+\frac{1}{N+\bar{N}} L_{-1} L_{1}\left|S_{B}\right\rangle-\frac{1}{N+\bar{N}} L_{-1} L_{-1}\left|B_{B}\right\rangle=0 \\
& \left|\mathcal{Z}_{2}\right\rangle=\frac{2(N+\bar{N}+3)}{N+\bar{N}+4} L_{0}\left|B_{B}\right\rangle-\frac{1}{N+\bar{N}+4} L_{-1} L_{1}\left|B_{B}\right\rangle+\frac{1}{N+\bar{N}+4} L_{1} L_{1}\left|S_{B}\right\rangle=0 \\
& \left|\mathcal{Z}_{3}\right\rangle=|\gamma\rangle=0
\end{aligned}
$$

Equations (5.7) match exactly the equation for the description of massless integer spin (2.8) and as in the previous case, equation (5.8) declares that the theory has a bosonic gauge invariant, auxiliary state. The corresponding Lagrangian is

$$
\begin{aligned}
\mathcal{L}= & \left\langle S_{B}\left|L_{0}\right| S_{B}\right\rangle-\left\langle S_{B}\left|\frac{1}{N+\bar{N}} L_{-1} L_{1}\right| S_{B}\right\rangle \\
& -\left\langle S_{B}\left|\frac{1}{N+\bar{N}} L_{-1} L_{-1}\right| B_{B}\right\rangle-\left\langle B_{B}\left|\frac{1}{N+\bar{N}+4} L_{1} L_{1}\right| S_{B}\right\rangle \\
& -2\left\langle B_{B}\left|\frac{N+\bar{N}+3}{N+\bar{N}+4} L_{0}\right| B_{B}\right\rangle+\left\langle B_{B}\left|\frac{1}{N+\bar{N}+4} L_{-1} L_{1}\right| B_{B}\right\rangle \\
& +\langle\gamma \mid \gamma\rangle .
\end{aligned}
$$

The fields $h_{\alpha(s) \dot{\alpha}(s)}, h_{\alpha(s-2) \dot{\alpha}(s-2)}$ correspond to the states $\left|S_{B}\right\rangle,\left|B_{B}\right\rangle$ and state $|\gamma\rangle$ will correspond to one of the supersymmetry auxiliary component. Unlike the previous case where we got the full spectrum of supersymmetric auxiliary components, this is no longer true in this case. In principle, we can allow $|\gamma\rangle$ to acquire some internal structure that can reveal the full set of bosonic auxiliary components, but at this approximation we can see them. That can happen if we demand from the very beginning for the theory to be off-shell supersymmetric and encode this information to the BRST charge by introducing the corresponding constraints that relate the bosonic and fermionic degrees of freedom. Of course, this will be automatic if we manage to repeat these type of construction in superspace. Finally, the most traditional way to detect the missing bosonic auxiliary components is to talk about the closure of the on-shell supersymmetry transformations. 


\section{Supersymmetric invariant theory}

In the previous two sections we have derived the spinorial BRST description of free massless higher spins. We noticed that the field that describes the $s+1 / 2$ spin satisfies the equations of motion of the field that describes the $s$ spin. Of course this was to be expected because the constraints that describe the integer spin $s$ are subset of the constraints that describe the half-integer spin $s+1 / 2$, but nevertheless it provides some reasoning to why one should expect the combination of these two systems to have supersymmetry. Also we have seen that the BRST approach is powerful enough, that even though we have not mentioned anything about supersymmetry, it naturally gives rise to components that will play the role of supersymmetric auxiliary fields, once we introduce supersymmetry.

\subsection{On-shell formulation}

The above observations, force us to discuss the supersymmetric theory. In this part, we will focus on the on-shell discussion. For that we consider the lagrangian

$$
\mathcal{L}_{S}=\mathcal{L}_{B}\left|+\mathcal{L}_{F}\right|
$$

where by $\mathcal{L}_{B} \mid$ we mean the bosonic lagrangian (5.9) with the auxiliary component $(|\gamma\rangle)$ integrated out. Similarly, $\mathcal{L}_{F} \mid$ is the fermionic lagrangian (4.13) without components $|\beta\rangle,|\rho\rangle$. It would be interesting to see how this formulation accommodates on-shell supersymmetry.

First of all, since we have to be able to map bosons to fermions and backwards, we must be able to change the parity of a state $|\Phi\rangle=\Phi_{\alpha(k) \dot{\alpha}(l)} a^{\dagger \alpha(k)} \bar{a}^{\dagger \dot{\alpha}(l)}|0, \overline{0}\rangle$. The parity of such a state is controlled by the total number of creation operators (free indices) it includes. States with $N+\bar{N}=$ even, have zero parity and states with $N+\bar{N}=$ odd, have parity one. ${ }^{7}$ Therefore, to convert a state from one parity to the other we have to change the value of $N+\bar{N}$ by a step of one. There is no object that can do this, therefore we introduce a parameter with an undotted index $\epsilon_{\alpha}\left(\epsilon_{\alpha} a^{\dagger \alpha}, \epsilon^{\beta} a_{\beta}\right)$ and its complex conjugate $\bar{\epsilon}_{\dot{\alpha}}\left(\bar{\epsilon}_{\dot{\alpha}} \bar{a}^{\dagger \dot{\alpha}}, \bar{\epsilon}^{\dot{\beta}} \bar{a}_{\dot{\beta}}\right)$

For the case of global supersymmetry these parameters are constants and we can prove that they satisfy the following identities

$$
\begin{array}{rlrl}
L_{1} \epsilon_{\alpha} a^{\dagger \alpha} & =(N+2) \epsilon_{\alpha} a^{\dagger \alpha} L_{1}+\frac{i}{N+1} \epsilon^{\beta} a_{\beta} T_{0}^{\prime}, & L_{-1} \epsilon_{\alpha} a^{\dagger \alpha} & =\epsilon_{\alpha} a^{\dagger \alpha} L_{-1}, \\
T_{0} \epsilon_{\alpha} a^{\dagger \alpha} & =(N+2) \epsilon_{\alpha} a^{\dagger \alpha} T_{0}+\frac{i}{N+1} \epsilon^{\beta} a_{\beta} L_{-1}, & T_{0}^{\prime} \epsilon_{\alpha} a^{\dagger \alpha}=\epsilon_{\alpha} a^{\dagger \alpha} T_{0}^{\prime}, \\
L_{-1} \epsilon^{\beta} a_{\beta} & =\frac{N}{(N+1)^{2}} \epsilon^{\beta} a_{\beta} L_{-1}+\frac{i}{N+1} \epsilon_{\alpha} a^{\dagger \alpha} T_{0}, & L_{1} \epsilon^{\beta} a_{\beta}=\epsilon^{\beta} a_{\beta} L_{1}, \\
T_{0}^{\prime} \epsilon^{\beta} a_{\beta} & =\frac{N}{(N+1)^{2}} \epsilon^{\beta} a_{\beta} T_{0}^{\prime}+\frac{i}{N+1} \epsilon_{\alpha} a^{\dagger \alpha} L_{1}, & T_{0} \epsilon^{\beta} a_{\beta}=\epsilon^{\beta} a_{\beta} T_{0}
\end{array}
$$

\footnotetext{
${ }^{7}$ A definition of parity in this context can be $\varepsilon=N+\bar{N}(\bmod 2)$.
} 


$$
\begin{array}{rlrl}
L_{1} \bar{\epsilon}_{\dot{\alpha}} \bar{a}^{\dagger \dot{\alpha}} & =(\bar{N}+2) \bar{\epsilon}_{\dot{\alpha}} \bar{a}^{\dagger \dot{\alpha}} L_{1}+\frac{i}{\bar{N}+1} \bar{\epsilon}^{\dot{\beta}} \bar{a}_{\dot{\beta}} T_{0}, & L_{-1} \bar{\epsilon}_{\dot{\alpha}} \bar{a}^{\dagger \dot{\alpha}}=\bar{\epsilon}_{\dot{\alpha}} \bar{a}^{\dagger \dot{\alpha}} L_{-1}, \\
T_{0}^{\prime} \bar{\epsilon}_{\dot{\alpha}} \bar{a}^{\dagger \dot{\alpha}}=(\bar{N}+2) \bar{\epsilon}_{\dot{\alpha}} \bar{a}^{\dagger \dot{\alpha}} T_{0}^{\prime}+\frac{i}{\bar{N}+1} \bar{\epsilon}^{\dot{\beta}} \bar{a}_{\dot{\beta}} L_{-1}, & T_{0} \bar{\epsilon}_{\dot{\alpha}} \bar{a}^{\dagger \dot{\alpha}}=\bar{\epsilon}_{\dot{\alpha}} \bar{a}^{\dagger \dot{\alpha}} T_{0}, \\
L_{-1} \bar{\epsilon}^{\dot{\beta}} \bar{a}_{\dot{\beta}}=\frac{\bar{N}}{(\bar{N}+1)^{2}} \bar{\epsilon}^{\dot{\beta}^{\beta}} \bar{a}_{\dot{\beta}} L_{-1}+\frac{i}{\bar{N}+1} \bar{\epsilon}_{\dot{\alpha}} \bar{a}^{\dagger \dot{\alpha}} T_{0}^{\prime}, & L_{1} \dot{\epsilon}^{\dot{\beta}} \bar{a}_{\dot{\beta}}=\bar{\epsilon}^{\dot{\beta}} \bar{a}_{\dot{\beta}} L_{1}, \\
T_{0} \bar{\epsilon}^{\dot{\beta}} \bar{a}_{\dot{\beta}}=\frac{\bar{N}}{(\bar{N}+1)^{2}} \bar{\epsilon}^{\dot{\beta}} \bar{a}_{\dot{\beta}} T_{0}+\frac{i}{\bar{N}+1} \bar{\epsilon}_{\dot{\alpha}} \bar{a}^{\dagger \dot{\alpha}} L_{1}, & T_{0}^{\prime} \bar{\epsilon}^{\dot{\beta}} \bar{a}_{\dot{\beta}}=\bar{\epsilon}^{\dot{\beta}} \bar{a}_{\dot{\beta}} T_{0}^{\prime}
\end{array}
$$

Now using (6.2) with the fermionic equations (4.9) we can prove the following

$$
\begin{aligned}
\left|\tilde{\mathcal{E}}_{1}\right\rangle & \equiv \epsilon^{\beta} a_{\beta}\left|\mathcal{E}_{1}\right\rangle=T_{0}\left|\tilde{S}_{B}\right\rangle-L_{-1}\left|\tilde{A}_{B}\right\rangle \\
\left|\tilde{\mathcal{E}}_{2}\right\rangle & \equiv \frac{N}{N+1} \epsilon^{\beta} a_{\beta}\left|\mathcal{E}_{2}\right\rangle-i(N+2)(\bar{N}+2) \epsilon_{\alpha} a^{\dagger \alpha}\left|\mathcal{E}_{3}\right\rangle \\
& =(N+\bar{N}+2) T_{0}^{\prime}\left|\tilde{A}_{B}\right\rangle+N(\bar{N}+1) L_{1}\left|\tilde{S}_{B}\right\rangle-(N+1)(\bar{N}+2) L_{-1}\left|\tilde{B}_{B}\right\rangle, \\
\left|\tilde{\mathcal{E}}_{3}\right\rangle & \equiv(N+4) \epsilon^{\beta} a_{\beta}\left|\mathcal{E}_{3}\right\rangle=T_{0}\left|\tilde{B}_{B}\right\rangle-L_{1}\left|\tilde{A}_{B}\right\rangle
\end{aligned}
$$

with

$$
\begin{aligned}
\left|\tilde{S}_{B}\right\rangle & =\epsilon^{\beta} a_{\beta}\left|S_{F}\right\rangle+\frac{i}{N} \epsilon_{\alpha} a^{\dagger \alpha}\left|A_{F}\right\rangle, \\
\left|\tilde{A}_{B}\right\rangle & =(N+3) \epsilon^{\beta} a_{\beta}\left|A_{F}\right\rangle, \\
\left|\tilde{B}_{B}\right\rangle & =(N+3) \epsilon^{\beta} a_{\beta}\left|B_{F}\right\rangle .
\end{aligned}
$$

The conclusion is that, the tilded equations are satisfied once the untilded equations are satisfied and also both sets have exactly the same structure. Therefore, states $\left|\tilde{S}_{B}\right\rangle,\left|\tilde{A}_{B}\right\rangle,\left|\tilde{B}_{B}\right\rangle$ on-shell satisfy the same equations as in (4.9) and therefore due to (4.12), the states $\left|\tilde{S}_{B}\right\rangle,\left|\tilde{B}_{B}\right\rangle$ satisfy exactly the integer spin equations (5.7). Hence, we can not distinguish between $\left(\left|S_{B}\right\rangle,\left|B_{B}\right\rangle\right)$ and $\left(\left|S_{B}\right\rangle+\left|\tilde{S}_{B}\right\rangle+\right.$ c.c., $\left|B_{B}\right\rangle+\left|\tilde{B}_{B}\right\rangle+$ c.c. $)$. Therefore, the integer spin theory is invariant under the transformation

$$
\begin{aligned}
\delta_{S}\left|S_{B}\right\rangle & =\epsilon^{\beta} a_{\beta}\left|S_{F}\right\rangle+\frac{i}{N} \epsilon_{\alpha} a^{\dagger \alpha}\left|A_{F}\right\rangle+c . c . \\
\delta_{S}\left|B_{B}\right\rangle & =(N+3) \epsilon^{\beta} a_{\beta}\left|B_{F}\right\rangle+c . c .
\end{aligned}
$$

Furthermore, if we start from the bosonic equations (5.7) and use (6.3) we can show that,

$$
\begin{aligned}
\left|\tilde{\mathcal{Z}}_{1}\right\rangle & \equiv \bar{\epsilon}_{\dot{\alpha}} \bar{a}^{\dagger \dot{\alpha}}\left|\mathcal{Z}_{1}\right\rangle=T_{0}\left|\tilde{S}_{F}\right\rangle-L_{-1}\left|\tilde{A}_{F}\right\rangle \\
\left|\tilde{\mathcal{Z}}_{2}\right\rangle & \equiv \frac{(N+2)(\bar{N}+1)}{N+3} \bar{\epsilon}_{\dot{\alpha}} \bar{a}^{\dagger \dot{\alpha}}\left|\mathcal{Z}_{2}\right\rangle=T_{0}\left|\tilde{B}_{F}\right\rangle-L_{1}\left|\tilde{A}_{F}\right\rangle \\
\left|\tilde{\mathcal{Z}}_{3}\right\rangle & \equiv i N \bar{\epsilon}^{\dot{\beta}} \bar{a}_{\dot{\beta}}\left|\mathcal{Z}_{1}\right\rangle \\
& =(N+\bar{N}+2) T_{0}^{\prime}\left|\tilde{A}_{F}\right\rangle+N(\bar{N}+1) L_{1}\left|\tilde{S}_{F}\right\rangle-(N+1)(\bar{N}+2) L_{-1}\left|\tilde{B}_{F}\right\rangle
\end{aligned}
$$


where

$$
\begin{aligned}
\left|\tilde{S}_{F}\right\rangle= & \frac{1}{N \bar{N}} \bar{\epsilon}_{\dot{\alpha}} \bar{a}^{\dagger \dot{\alpha}} T_{0}^{\prime}\left|S_{B}\right\rangle \\
\left|\tilde{A}_{F}\right\rangle= & -\frac{(N+1)(\bar{N}-1)}{(N+\bar{N}+1)(N+2) \bar{N}} \bar{\epsilon}_{\dot{\alpha}} \bar{a}^{\dagger \dot{\alpha}} L_{1}\left|S_{B}\right\rangle+\frac{1}{N+\bar{N}+1} \bar{\epsilon}_{\dot{\alpha}} \bar{a}^{\dagger \dot{\alpha}} L_{-1}\left|B_{B}\right\rangle, \\
\left|\tilde{B}_{F}\right\rangle= & \frac{\bar{N}+2}{(N+2)(\bar{N}+1)} \bar{\epsilon}_{\dot{\alpha}} \bar{a}^{\dagger \dot{\alpha}} T_{0}^{\prime}\left|B_{B}\right\rangle+i \frac{\bar{N}+2}{(\bar{N}+1)(N+\bar{N}+3)} \bar{\epsilon}^{\dot{\beta}} \bar{a}_{\dot{\beta}} L_{-1}\left|B_{B}\right\rangle \\
& -i \frac{N+1}{(N+2)(N+\bar{N}+3)} \bar{\epsilon}^{\dot{\beta}} \bar{a}_{\dot{\beta}} L_{1}\left|S_{B}\right\rangle .
\end{aligned}
$$

The result is that states $\left|\tilde{S}_{F}\right\rangle,\left|\tilde{A}_{F}\right\rangle,\left|\tilde{B}_{F}\right\rangle$ on-shell satisfy equations (4.9) and the theory of half-integer spins is invariant under the transformations

$$
\begin{aligned}
\delta_{S}\left|S_{F}\right\rangle= & \frac{1}{N \bar{N}} \bar{\epsilon}_{\dot{\alpha}} \bar{a}^{\dagger \dot{\alpha}} T_{0}^{\prime}\left|S_{B}\right\rangle, \\
\delta_{S}\left|A_{F}\right\rangle= & -\frac{(N+1)(\bar{N}-1)}{(N+\bar{N}+1)(N+2) \bar{N}} \bar{\epsilon}_{\dot{\alpha}} \bar{a}^{\dagger \dot{\alpha}} L_{1}\left|S_{B}\right\rangle+\frac{1}{N+\bar{N}+1} \bar{\epsilon}_{\dot{\alpha}} \bar{a}^{\dagger \dot{\alpha}} L_{-1}\left|B_{B}\right\rangle, \\
\delta_{S}\left|B_{F}\right\rangle= & \frac{\bar{N}+2}{(N+2)(\bar{N}+1)} \bar{\epsilon}_{\dot{\alpha}} \bar{a}^{\dagger \dot{\alpha}} T_{0}^{\prime}\left|B_{B}\right\rangle+i \frac{\bar{N}+2}{(\bar{N}+1)(N+\bar{N}+3)} \bar{\epsilon}^{\dot{\beta}} \bar{a}_{\dot{\beta}} L_{-1}\left|B_{B}\right\rangle \\
& -i \frac{N+1}{(N+2)(N+\bar{N}+3)} \bar{\epsilon}^{\dot{\beta}} \bar{a}_{\dot{\beta}} L_{1}\left|S_{B}\right\rangle .
\end{aligned}
$$

Transformations (6.6), (6.9) are exactly the on-shell supersymmetry transformations for massless integer superspin [19,20]. Similar calculations can be done to demonstrate that there is another transformation

$$
\begin{aligned}
\delta\left|S_{B}\right\rangle= & \bar{\epsilon}_{\dot{\alpha}} \bar{a}^{\dagger \dot{\alpha}}\left|S_{F}\right\rangle \\
\delta\left|B_{B}\right\rangle= & \frac{(\bar{N}+2)^{2}}{\bar{N}+1} \bar{\epsilon}_{\dot{\alpha}} \bar{a}^{\dagger \dot{\alpha}}\left|B_{F}\right\rangle+i \frac{\bar{N}+2}{\bar{N}+1} \bar{\epsilon}^{\dot{\beta}} \bar{a}_{\dot{\beta}}\left|A_{F}\right\rangle \\
\delta\left|S_{F}\right\rangle= & \frac{1}{(N+1)(\bar{N}+1)} \epsilon^{\beta} a_{\beta} T_{0}^{\prime}\left|S_{B}\right\rangle-i \frac{\bar{N}}{(N+\bar{N}+1)(N+1)(\bar{N}+1)} \epsilon_{\alpha} a^{\dagger \alpha} L_{1}\left|S_{B}\right\rangle \\
& +\frac{i}{(N+\bar{N}+1) N} \epsilon_{\alpha} a^{\dagger \alpha} L_{-1}\left|B_{B}\right\rangle \\
\delta\left|A_{F}\right\rangle= & -\frac{(N+2) \bar{N}}{(N+\bar{N}+3)(\bar{N}+1)} \epsilon^{\beta} a_{\beta} L_{1}\left|S_{B}\right\rangle+\frac{N+3}{N+\bar{N}+3} \epsilon^{\beta} a_{\beta} L_{-1}\left|B_{B}\right\rangle \\
\delta\left|B_{F}\right\rangle= & \frac{1}{\bar{N}+3} \epsilon^{\beta} a_{\beta} T_{0}^{\prime}\left|B_{B}\right\rangle .
\end{aligned}
$$

which corresponds to the on-shell supersymmetry transformations for massless half-integer superspin.

\subsection{Off-shell formulation}

In this subsection we discuss a possibility to construct off-shell supersymmetric Lagrangian formulation in the framework of the BRST construction. First of all, one updates the information of the BRST charge regarding supersymmetry. We have seen that for $\mathcal{N}=0$, 
BRST can give us part of the off-shell supersymmetric spectrum. However, we can see that in principle there is room for the extra supersymmetric auxiliary components to appear.

In the case of half-ineger spin, the auxiliary component $|\rho\rangle$ appeared in the lagrangian (4.13) as the lagrange multiplier in order to generate the equation $|\beta\rangle=0$. But we could have seen the existance of $|\rho\rangle$ as a redefinition of state $\left|A_{F}\right\rangle$ to $\left|A_{F}\right\rangle+|\rho\rangle$ in (4.2). The equation of motion and the gauge transformation of $\left|A_{F}\right\rangle$ would remain the same if $|\rho\rangle=0$ and $\delta_{G}|\rho\rangle=0$, exactly as predicted by lagrangian (4.13).

For the integer spin case, the $\mathcal{N}=0$ BRST construction gives rise to one auxiliary component $|\gamma\rangle$. It is gauge invariant and has mass dimensions two. Therefore, all the bosonic auxiliary components in the various supersymmetric theories will reside in it and can be viewed as components of

$$
|\gamma\rangle \sim|A\rangle+|u\rangle+|v\rangle+|S\rangle+|P\rangle+|U\rangle
$$

a deeper internal structure that has knowledge of supersymmetry. For consistency, they must be gauge invariant and vanish on-shell, i.e exactly as they behave in the supersymmetric theory. At the lagrangian description, the $\langle\gamma \mid \gamma\rangle$ in (5.9) will be replaced by the sum of the diagonal terms $\eta_{A}\langle A \mid A\rangle+\eta_{u}\langle u \mid u\rangle+\eta_{v}\langle v \mid v\rangle+\eta_{S}\langle S \mid S\rangle+\eta_{P}\langle P \mid P\rangle+\eta_{U}\langle U \mid U\rangle$. The phases $\eta$ will have to be chosen such that they match their correct relative signs. For example in the integer superspin case the corresponding bosonic part of the lagrangian will be

$$
\begin{aligned}
\mathcal{L}_{B}= & \left\langle S_{B}\left|L_{0}\right| S_{B}\right\rangle-\left\langle S_{B}\left|\frac{1}{N+\bar{N}} L_{-1} L_{1}\right| S_{B}\right\rangle \\
& -\left\langle S_{B}\left|\frac{1}{N+\bar{N}} L_{-1} L_{-1}\right| B_{B}\right\rangle-\left\langle B_{B}\left|\frac{1}{N+\bar{N}+4} L_{1} L_{1}\right| S_{B}\right\rangle \\
& -2\left\langle B_{B}\left|\frac{N+\bar{N}+3}{N+\bar{N}+4} L_{0}\right| B_{B}\right\rangle+\left\langle B_{B}\left|\frac{1}{N+\bar{N}+4} L_{-1} L_{1}\right| B_{B}\right\rangle \\
& -\langle A \mid A\rangle+\langle u \mid u\rangle+\langle v \mid v\rangle-\langle S \mid S\rangle-\langle P \mid P\rangle-\langle U \mid U\rangle
\end{aligned}
$$

and for the two half-integer superspins

$$
\begin{aligned}
\mathcal{L}_{B}^{\perp}= & \left\langle S_{B}\left|L_{0}\right| S_{B}\right\rangle-\left\langle S_{B}\left|\frac{1}{N+\bar{N}} L_{-1} L_{1}\right| S_{B}\right\rangle \\
& -\left\langle S_{B}\left|\frac{1}{N+\bar{N}} L_{-1} L_{-1}\right| B_{B}\right\rangle-\left\langle B_{B}\left|\frac{1}{N+\bar{N}+4} L_{1} L_{1}\right| S_{B}\right\rangle \\
& -2\left\langle B_{B}\left|\frac{N+\bar{N}+3}{N+\bar{N}+4} L_{0}\right| B_{B}\right\rangle+\left\langle B_{B}\left|\frac{1}{N+\bar{N}+4} L_{-1} L_{1}\right| B_{B}\right\rangle \\
& +\langle A \mid A\rangle+\langle u \mid u\rangle-\langle v \mid v\rangle-\langle S \mid S\rangle-\langle P \mid P\rangle+\langle U \mid U\rangle \\
\mathcal{L}_{B}^{\|}= & \left\langle S_{B}\left|L_{0}\right| S_{B}\right\rangle-\left\langle S_{B}\left|\frac{1}{N+\bar{N}} L_{-1} L_{1}\right| S_{B}\right\rangle \\
& -\left\langle S_{B}\left|\frac{1}{N+\bar{N}} L_{-1} L_{-1}\right| B_{B}\right\rangle-\left\langle B_{B}\left|\frac{1}{N+\bar{N}+4} L_{1} L_{1}\right| S_{B}\right\rangle \\
& -2\left\langle B_{B}\left|\frac{N+\bar{N}+3}{N+\bar{N}+4} L_{0}\right| B_{B}\right\rangle+\left\langle B_{B}\left|\frac{1}{N+\bar{N}+4} L_{-1} L_{1}\right| B_{B}\right\rangle \\
& +\langle A \mid A\rangle-\langle u \mid u\rangle-\langle v \mid v\rangle-\langle S \mid S\rangle+\langle P \mid P\rangle-\langle U \mid U\rangle .
\end{aligned}
$$


Also keep in mind that the value of the counting operators $N, \bar{N}$ as they act on these states will be different, meaning that they have different index structure depending the system we describe. But this piece of information is encoded in the oscillators expansion of the Fock state.

\section{Generalization for massive higher spin supersymmetric theories}

Here we briefly describe a possible way to generalized the BRST construction, described above, for massive supersymmetric higher spin theories.

For the massive case, the set of constraints that define the irreducible representations are modified in the following way:

- For half integer spin $(s+1 / 2)$ we must have

$$
\partial^{\beta \dot{\beta}} \psi_{\beta \alpha(s) \dot{\beta} \dot{\alpha}(s-1)}=0, i \partial_{\dot{\alpha}_{s+1}}^{\beta} \psi_{\beta \alpha(s) \dot{\alpha}(s)}+m \bar{\psi}_{\alpha(s) \dot{\alpha}(s+1)}=0
$$

- For integer spin $(s)$ we must have

$$
\partial^{\beta \dot{\beta}} h_{\beta \alpha(s-1) \dot{\beta} \dot{\alpha}(s-1)}=0,\left(\square-m^{2}\right) h_{\alpha(s) \dot{\alpha}(s)}=0 .
$$

It is known that Lagrangians that describe the two these systems are more complicate and involve a tower of real tensors of increasing rank $\left\{h, h_{a \dot{\alpha}}, h_{\alpha(2) \dot{\alpha}(2)}, \ldots, h_{\alpha(s-2) \dot{\alpha}(s-2)}\right.$, $\left.h_{\alpha(s) \dot{\alpha}(s)}\right\}$ for the integer spin case and a similar tower of tensors of increasing rank $\left\{\left(\psi_{\alpha}, \chi_{\alpha}\right),\left(\psi_{\alpha(2) \dot{\alpha}}, \chi_{\alpha(2) \dot{\alpha}}\right), \ldots,\left(\psi_{\alpha(s-1) \dot{\alpha}(s-2)}, \chi_{\alpha(s-1) \dot{\alpha}(s-2)}\right), \psi_{\alpha(s) \dot{\alpha}(s-1)}, \psi_{\alpha(s+1) \dot{\alpha}(s)}\right\}$ for the half integer case. A physical way to understand why this is the case is to view the $2 s+1$ states of spin $s$ as a collection of massless spin states with helicity 0 up to helicity $s$ and similarly the $2 s+2$ states of massive spin $s+1 / 2$ as a collection of massless spin states with helicity $1 / 2$ up to $s+1 / 2$.

To get the BRST description for the massive theory we have to follow similar steps as before, like converting all the above constraints into Fock space operators and calculate their algebra. Such a procedure could be done for integer spin, but for the half integer spin there is an obstacle. The Dirac equation, in this notation involves two states $(|\psi\rangle$ and $|\bar{\psi}\rangle)$, therefore it can not be expressed as a single Fock operator acting on one Fock state. It looks like, we have to involve both subalgebras $\left\{T_{0}, L_{1}, L_{-1}, L_{0}, N, \bar{N}\right\}$, $\left\{T_{0}^{\prime}, L_{1}, L_{-1}, L_{0}, N, \bar{N}\right\}$ and construct their direct sum. A possible way of doing that is to define a fermionic BRST charge in the following way

$$
Q \sim\left(\begin{array}{cc}
Q_{F} & \\
& \bar{Q}_{F}
\end{array}\right), \quad|\psi\rangle \sim\left(\begin{array}{c}
\left|\psi_{F}\right\rangle \\
\left|\bar{\psi}_{F}\right\rangle
\end{array}\right) .
$$

If that is the case, then the extension to the massive case would correspond to the existence of off-diagonal elements proportional to mass $m$.

Following this idea, we see that (7.1) and its complex conjugate can be written in the following way

$$
\left(\begin{array}{cc}
\partial^{\beta \dot{\beta}} & 0 \\
0 & \partial^{\beta \dot{\beta}}
\end{array}\right)\left(\begin{array}{l}
\psi_{\beta \alpha(s) \dot{\beta} \dot{\alpha}(s-1)} \\
\bar{\psi}_{\beta \alpha(s-1) \dot{\beta} \dot{\alpha}(s)}
\end{array}\right)=0, \quad\left(\begin{array}{cc}
i \partial^{\alpha_{s+1}} \dot{\alpha}_{s+1} & m \\
m & i \partial_{\alpha_{s+1}} \\
\dot{\alpha}_{s+1}
\end{array}\right)\left(\begin{array}{l}
\psi_{\alpha(s+1) \dot{\alpha}(s)} \\
\bar{\psi}_{\alpha(s) \dot{\alpha}(s+1)}
\end{array}\right)=0
$$


and therefore we define,

$$
\begin{array}{rlrl}
\hat{T}_{0} & =\left(\begin{array}{cc}
T_{0} & m \\
m & T_{0}^{\prime}
\end{array}\right), & \hat{L}_{0} & =\left(\begin{array}{cc}
L_{0}-m^{2} & 0 \\
0 & L_{0}-m^{2}
\end{array}\right) \\
\hat{L}_{1} & =\left(\begin{array}{cc}
L_{1} & 0 \\
0 & L_{1}
\end{array}\right), & \hat{L}_{-1}=\left(\begin{array}{cc}
L_{-1} & 0 \\
0 & L_{-1}
\end{array}\right)
\end{array}
$$

The rest of the procedure will follow the method developed earlier for BRST construction in massive higher spin theories [42-44]. It means that we have to get an extension of the algebra (3.6) with mass terms playing the role of central charges and therefore second class constraints. The conversion of the second class constraints due to mass, to first class will be done in the usual way by expanding our Fock space with more oscillators that will lead to the presence of the extra states required by the Lagrangian description. The exact construction will be investigated in another letter.

\section{Problem of superfield BRST construction}

Here we briefly describe a possible way to formulate the BRST construction for $\mathcal{N}=1$ supersymmetric higher spin theories in superfield form and discuss the difficulties of such an approach.

The irreducible representations of Super-Poincare group have been are defined by the following constraints

$$
\mathrm{D}^{\beta} H_{\beta \alpha(s-1) \dot{\alpha}(s)}=0, \overline{\mathrm{D}}^{\dot{\beta}} H_{\alpha(s) \dot{\beta} \dot{\alpha}(s-1)}=0, \mathrm{D}^{\gamma} \overline{\mathrm{D}}^{2} \mathrm{D}_{\gamma} H_{\alpha(s) \dot{\alpha}(s)}=0
$$

for massless, half-integer superspin where $H_{\alpha(s) \dot{\alpha}(s)}$ is a real bosonic superfield and

$$
\mathrm{D}^{\beta} \Psi_{\beta \alpha(s) \dot{\alpha}(s-1)}=0, \overline{\mathrm{D}}^{\dot{\beta}} \Psi_{\alpha(s+1) \dot{\beta} \dot{\alpha}(s-1)}, \mathrm{D}^{\beta} \overline{\mathrm{D}}_{\left(\dot{\alpha}_{s+1}\right.} \Psi_{\beta \alpha(s) \dot{\alpha}(s))}=0
$$

for massless, integer superspin. The extension of these for the massive irreps can be done by simply adding the mass term to the appropriate equations. In the same spirit, we should define the corresponding Fock space operators

$$
L_{1}=\mathrm{D}^{\beta} a_{\beta}, L_{2}=\overline{\mathrm{D}}^{\dot{\beta}} \bar{a}_{\dot{\beta}}, L_{-2}=\mathrm{D}_{\alpha} a^{\dagger \alpha}, L_{-1}=\overline{\mathrm{D}}_{\dot{\alpha}} \bar{a}^{\dagger \dot{\alpha}}, \ldots
$$

calculate the algebra and attempt to build a BRST charge. However, it is not obvious if the algebra closes or keeps generating increasing powers of $\square$ and whether it must be interpreted as a nonlinear algebra or not. Furthermore, it is not obvious how to choose the vacuum state such that, we generate exactly the desired equations of motion. Also, we have to consider the possibility to introduce matrix like operators in order to match the component discussion.

The simplest gauge invariant theory in superspace is the massless vector multiplet. The equation of motion is

$$
\mathrm{D}^{\gamma} \overline{\mathrm{D}}^{2} \mathrm{D}_{\gamma} H=0
$$


and it is invariant under the transformation

$$
\delta H=\mathrm{D}^{2} \bar{L}-\overline{\mathrm{D}}^{2} L .
$$

Therefore, the BRST charge must include the operators $\mathrm{D}^{2}, \overline{\mathrm{D}}^{2}, \mathrm{D}^{\gamma} \overline{\mathrm{D}}^{2} \mathrm{D}_{\gamma}$ which have the algebra

$$
\left[\mathrm{D}^{\gamma} \overline{\mathrm{D}}^{2} \mathrm{D}_{\gamma}, \mathrm{D}^{2}\right]=\left[\mathrm{D}^{\gamma} \overline{\mathrm{D}}^{2} \mathrm{D}_{\gamma}, \overline{\mathrm{D}}^{2}\right]=0,\left[\mathrm{D}^{2}, \mathrm{D}^{2}\right]=-\frac{i}{2} \partial^{\alpha \dot{\alpha}}\left[\mathrm{D}_{\alpha}, \overline{\mathrm{D}}_{\dot{\alpha}}\right]
$$

To close the algebra, without introducing operators with higher rank or make it non-linear, we add to our list of operators $\Delta=\left[\mathrm{D}^{2}, \mathrm{D}^{2}\right]$ and we attempt the construction of a BRST charge $Q=\eta D^{\gamma} \overline{\mathrm{D}}^{2} \mathrm{D}_{\gamma}+\zeta \mathrm{D}^{2}+\xi \overline{\mathrm{D}}^{2}+\rho\left[\mathrm{D}^{2}, \overline{\mathrm{D}}^{2}\right]+c$. In order $Q$ to be nilpotent we must satisfy the following:

$$
\begin{aligned}
\eta^{2}=c^{2}=\rho^{2} & =0, \\
\{\eta, c\}=\{\zeta, c\}=\{\xi, c\}=\{\zeta, \xi\} & =0, \\
{[\rho, \zeta]=[\rho, \xi] } & =0, \\
\zeta \xi+\{\rho, c\} & =0 .
\end{aligned}
$$

Notice that $\zeta$ and $\xi$ do not have to be nilpotent and also $\rho$ has to commute with them. A solution of them is to have $\rho=-\zeta \xi P_{c}$ where $\left\{c, P_{c}\right\}=1$, but we can not find a choice of vacuum that will generate equation (8.4).

An observation is that various superspace operators (like $\mathrm{D}^{2}$ and $\overline{\mathrm{D}}^{2}$ ) have by themselves the nilpotent property hence, they do not require the existence of a nilpotent ghost in the corresponding BRST charge. Another observation is that, unlike the spacetime constructions where the nature of the constraints is always the same, in superspace depending on the number of covariant derivatives we will have even or odd parity operators therefore we need to have both fermionic and bosonic ghost like oscillators.

The construction of a BRST charge for superspace, is unclear yet. A possible direction is to look for a different but equivalent set of constraints to define the irreducible representations in a way that allows a direct application of the known methods. Another way, is to consider the possibility of generalized charges where the kernel of $Q$ does not include the image of $Q$. This will relax the nilpotent condition to a more general requirement for gauge invariance $Q \cdot Q^{\prime}=0$, where $Q$ is responsible for the equations of motion and $Q^{\prime}$ for the gauge transformations.

\section{Summary}

We have presented the BRST approach to Lagrangian description of $4 \mathrm{D}, \mathcal{N}=1$ supersymmetric free massless higher spin theories using the spinorial index notation. Generalization of the BRST construction for the supersymmetric higher spin models was given and the supersymmetric higher spin field Lagrangian was obtained.

We have demonstrated that the BRST description of massless free higher spins in the 2component spinor notation is preferable because it circumvents the second class constraints related with the trace and $\gamma$-trace conditions in the definition of higher spins. 
Furthermore, we have shown that the BRST procedure not only provides all the necessary auxiliary fields for the higher spin description but also give rise to extra auxiliary components that play the role of off-shell supersymmetric auxiliary components. In the other words, one can expect that the BRST approach will give a possibility of complete off-shell Lagrangian formulation of higher spin supersymmetric models. A hint for that is lagrangian (4.13) which matches exactly the fermionic part of the corresponding superspace lagrangians that describe integer and half-integer superspin irreducible representations of the super-Poincaré group.

Finally, we have illustrated how, on-shell supersymmetry transformations emerge just because, the algebra of the bosonic constraints is included in the algebra of the fermionic constraints.

All the above results motivates us to carry out a similar analysis for the massive higher spin supersymmetric models and also for superspace formulated theories. In the first case, it seems that we have to expend our Fock space, so that for every state we introduce its complex conjugate. That forces us to redefine the various operators of the massless theory in terms of matrices acting on a complex conjugated enhanced Fock vector. Afterwards, we have to calculate the algebra of these objects and check a) if the usual BRST procedure can apply and b) if there is a vacuum state that will give the desired equations of motion. Also, we can apply the methods developed earlier for BRST construction in massive higher spin theories [42-44]. For the superspace case, the picture is not so clear regarding the closure of the algebra of the constraints and its peculiarities like the fact that it already includes nilpotent objects. We plan to study all these aspects in the forthcoming works.

When all the above are understood and we have a good understanding of the free supersymmetric theories viewed from the BRST point of view, we can start asking questions about interactions. This will correspond to deforming the BRST charge by adding vertex operators that result into interactions among the various Fock space vectors. This will be an even more challenging problem because not only we will have to face the difficulties of interacting higher spins but we want to do it in a supersymmetric way.

\section{Acknowledgments}

The authors are very grateful to V.A. Krykhtin and W.D. Linch for helpful discussions. K.K. is thankful to Center of Theoretical Physics at Tomsk State Pedagogical University for warm hospitality. The work of I.L.B was supported by the RFBR grant, project No 15-02-03594 and by the Ministry of Education and Science of Russian Federation, project 3.967.2014/K. The work of K.K was supported by RFBR grant, project No 15-32-50086 and by P201/12/G028 of the Grant agency of the Czech Republic.

\section{A Two-component spinor notation}

Consider the 4D Minkowski, Clifford algebra

$$
\left\{e_{m}, e_{n}\right\}=2 \eta_{m n} .
$$


It is well know, that the objects

$$
J_{m n}=-\frac{i}{4}\left[e_{m}, e_{n}\right], P_{m}=e_{m}
$$

satisfy the Poincaré algebra and therefore the representations of the Poincaré algebra can be generated by the representations of the Clifford algebra. Also, it is well known that in $4 \mathrm{D}$ we can have Weyl spinors, thus the smallest representations of Clifford algebra are the left $\psi_{\alpha}$ and right $\chi^{\dot{\alpha}}$ spinors. Under the action of the Poincare algebra they transform in the following way:

$$
\left[J_{m n}, \psi_{\alpha}\right]=i\left(\sigma_{m n}\right)_{\alpha}{ }^{\beta} \psi_{\beta},\left[J_{m n}, \chi^{\dot{\alpha}}\right]=i\left(\bar{\sigma}_{m n}\right)_{\dot{\beta}}^{\dot{\alpha}} \chi^{\dot{\beta}}
$$

where

$$
\begin{aligned}
\left(\sigma_{m n}\right)_{\alpha}{ }^{\beta} & =\frac{1}{4}\left(\sigma_{m}\right)_{\alpha \dot{\alpha}}\left(\bar{\sigma}_{n}\right)^{\dot{\alpha} \beta}-\frac{1}{4}\left(\sigma_{n}\right)_{\alpha \dot{\alpha}}\left(\bar{\sigma}_{m}\right)^{\dot{\alpha} \beta}, \\
\left(\bar{\sigma}_{m n}\right)_{\dot{\beta}}^{\dot{\alpha}} & =\frac{1}{4}\left(\bar{\sigma}_{m}\right)^{\dot{\alpha} \alpha}\left(\sigma_{n}\right)_{\alpha \dot{\beta}}-\frac{1}{4}\left(\bar{\sigma}_{n}\right)^{\dot{\alpha} \alpha}\left(\sigma_{m}\right)_{\alpha \dot{\beta}} .
\end{aligned}
$$

The $\sigma_{m}=(1, \vec{\sigma})$ and $\bar{\sigma}_{m}=(-1, \vec{\sigma}),{ }^{8}$ satisfy a list of very useful properties

$$
\begin{aligned}
\left(\sigma_{m}\right)_{\alpha \dot{\alpha}}\left(\bar{\sigma}_{n}\right)^{\dot{\alpha} \beta}+\left(\sigma_{n}\right)_{\alpha \dot{\alpha}}\left(\bar{\sigma}_{m}\right)^{\dot{\alpha} \beta} & =2 \eta_{m n} \delta_{\alpha}{ }^{\beta}, \\
\left(\overline{\sigma_{m}}\right)^{\dot{\alpha} \alpha}\left(\sigma_{n}\right)_{\alpha \dot{\beta}}+\left(\bar{\sigma}_{n}\right)^{\dot{\alpha} \alpha}\left(\sigma_{m}\right)_{\alpha \dot{\beta}} & =2 \eta_{m n} \delta^{\dot{\alpha}} \dot{\beta}, \\
\left(\sigma^{m}\right)_{\alpha \dot{\alpha}}\left(\bar{\sigma}_{m}\right)^{\dot{\beta} \beta} & =2 \delta_{\alpha}{ }^{\beta} \delta_{\dot{\alpha}}^{\dot{\beta}}, \\
\frac{1}{2} \epsilon^{k l m n} \sigma_{m n} & =-i \sigma^{k l}, \quad \frac{1}{2} \epsilon^{k l m n} \bar{\sigma}_{m n}=i \bar{\sigma}^{k l}, \\
\sigma^{k} \bar{\sigma}^{l n} & =\frac{1}{2} \eta^{l k} \sigma^{n}-\frac{1}{2} \eta^{n k} \sigma^{l}-\frac{i}{2} \epsilon^{k l n m} \sigma_{m}, \\
\bar{\sigma}^{k} \sigma^{l n} & =\frac{1}{2} \eta^{l k} \bar{\sigma}^{n}-\frac{1}{2} \eta^{n k} \bar{\sigma}^{l}+\frac{i}{2} \epsilon^{k l n m} \bar{\sigma}_{m}, \\
\sigma^{l n} \sigma^{k} & =-\frac{1}{2} \eta^{l k} \sigma^{n}+\frac{1}{2} \eta^{n k} \sigma^{l}-\frac{i}{2} \epsilon^{k l n m} \sigma_{m}, \\
\bar{\sigma}^{l n} \bar{\sigma}^{k} & =-\frac{1}{2} \eta^{l k} \bar{\sigma}^{n}+\frac{1}{2} \eta^{n k} \bar{\sigma}^{l}+\frac{i}{2} \epsilon^{k l n m} \bar{\sigma}_{m} .
\end{aligned}
$$

The $\left(\sigma_{m}\right)_{\alpha \dot{\alpha}},\left(\bar{\sigma}_{m}\right)^{\dot{\alpha} \alpha}$ are the only objects that have all three kinds of indices. For this reason they are used for converting vector indices to left-right indices and vice versa. For example:

$$
A_{m}=\left(\bar{\sigma}_{m}\right)^{\dot{\alpha} \alpha} A_{\alpha \dot{\alpha}}, A_{\alpha \dot{\alpha}}=\frac{1}{2}\left(\sigma^{m}\right)_{\alpha \dot{\alpha}} A_{m} .
$$

An example of that would be the partial derivative, $\partial_{m}$. So let's define

$$
\partial_{\alpha \dot{\alpha}}=\left(\sigma^{m}\right)_{\alpha \dot{\alpha}} \partial_{m} \text {. }
$$

We can show that it has the properties

$$
\begin{aligned}
\partial^{\alpha \dot{\alpha}} \partial_{\alpha \dot{\beta}}=\delta^{\dot{\alpha}}{ }_{\dot{\beta}} \square, \quad \partial^{\dot{\alpha} \alpha} \partial_{\alpha \dot{\beta}}=\delta^{\dot{\alpha}}{ }_{\dot{\beta}} \square \\
\partial^{\alpha}{ }_{\dot{\beta}} \partial_{\beta}{ }^{\dot{\alpha}}-\partial^{\alpha \dot{\alpha}} \partial_{\beta \dot{\beta}}=\delta^{\alpha}{ }_{\beta} \delta^{\dot{\alpha}}{ }_{\dot{\beta}}
\end{aligned}
$$

\footnotetext{
${ }^{8} \vec{\sigma}$ are the three Pauli matrices.
} 
The conversion of vector indices to spinorial ones, doesn't work just for vectors but for higher rank tensors as well. For example consider the case of a rank two tensor

$$
A_{m n}=\left(\bar{\sigma}_{m}\right)^{\dot{\alpha} \alpha}\left(\bar{\sigma}_{n}\right)^{\dot{\beta} \beta} A_{\alpha \beta \dot{\alpha} \dot{\beta}}
$$

$A_{\alpha \beta \dot{\alpha} \dot{\beta}}$ can be further decomposed by symmetrizing and anti-symmetrizing the undotted and dotted pair of indices.

$$
A_{\alpha \beta \dot{\alpha} \dot{\beta}}=A_{\alpha \beta \dot{\alpha} \dot{\beta}}^{(S, S)}+C_{\alpha \beta} A_{\dot{\alpha} \dot{\beta}}^{(A, S)}+C_{\dot{\alpha} \dot{\beta}} A_{\alpha \dot{\beta}}^{(S, A)}+C_{\alpha \beta} C_{\dot{\alpha} \dot{\beta}} A^{(A, A)}
$$

and we get that

$$
\begin{aligned}
A_{\alpha \beta \dot{\alpha} \dot{\beta}}^{(S, S)} & =\frac{1}{16}\left(\sigma^{m}\right)_{(\alpha(\dot{\alpha}}\left(\sigma^{n}\right)_{\beta) \dot{\beta})} A_{m n} \\
A_{\dot{\alpha} \dot{\beta}}^{(A, S)} & =-\frac{1}{4}\left(\bar{\sigma}^{m n}\right)_{\dot{\alpha} \dot{\beta}} A_{m n} \\
A_{\alpha \beta}^{(S, A)} & =\frac{1}{4}\left(\sigma^{m n}\right)_{\alpha \beta} A_{m n} \\
A^{(A, A)} & =\frac{1}{8} \eta^{m n} A_{m n}
\end{aligned}
$$

From the above we can see that for a rank two antisymmetric tensor (like the generators of the Lorentz group, $\left.\mathcal{J}_{m n}\right)$ the completely symmetric and the scalar terms vanish $\left(A_{\alpha \beta \dot{\alpha} \dot{\beta}}^{(S, S)}=\right.$ $\left.0, A^{(A, A)}=0\right)$ and we get

$$
\mathcal{J}_{m n}=2\left(\sigma_{m n}\right)^{\alpha \beta} \mathcal{J}_{\alpha \beta}-2\left(\bar{\sigma}_{m n}\right)^{\dot{\alpha} \dot{\beta}} \mathcal{J}_{\dot{\alpha} \dot{\beta}}
$$

The $C_{\alpha \beta}$ and $C_{\dot{\alpha} \dot{\beta}}$ are antisymmetric objects defined in the following way

$$
C^{\alpha \beta}=\left(\begin{array}{cc}
0 & i \\
-i & 0
\end{array}\right)=C^{\dot{\alpha} \dot{\beta}}, \quad C_{\alpha \beta}=\left(\begin{array}{cc}
0 & -i \\
i & 0
\end{array}\right)=C_{\dot{\alpha} \dot{\beta}}
$$

and they are also used as a metric to raise and lower the spinorial indices. They have the property

$$
\begin{aligned}
& C^{\alpha \beta} C_{\gamma \rho}=\delta^{\alpha}{ }_{\gamma} \delta^{\beta}{ }_{\rho}-\delta^{\alpha}{ }_{\rho} \delta^{\beta}{ }_{\gamma}, \\
& C^{\dot{\alpha} \dot{\beta}} C_{\dot{\gamma} \dot{\rho}}=\delta^{\dot{\alpha}}{ }_{\dot{\gamma}} \delta^{\dot{\beta}}{ }_{\dot{\rho}}-\delta^{\dot{\alpha}}{ }_{\dot{\rho}} \delta^{\dot{\beta}} \dot{\gamma} .
\end{aligned}
$$

For the description of higher spins we will use fields $\phi$ with $k$ number of undotted indices and $l$ number of dotted indices and have the property that are independently symmetrized in both of them. We will denote that by writing $\phi_{\alpha(k) \dot{\alpha}(l)}$. Then from (A.5), (A.10), (A.11) follows that the trace and $\gamma$-trace of these fields vanish identically. That is very useful because in this formulation of higher spins, the trace constraints that usually introduce second class constraints in the BRST approach are not present any more.

Open Access. This article is distributed under the terms of the Creative Commons Attribution License (CC-BY 4.0), which permits any use, distribution and reproduction in any medium, provided the original author(s) and source are credited. 


\section{References}

[1] J. Wess and J. Bagger, Supersymmetry and Supergravity, Princeton University Press, Princeton U.S.A. (1983).

[2] P. West, Introduction to Supersymmetry and Supergravity, World Scientific, Singapore (1986).

[3] A.S. Galperin, E.A. Ivanov, V.I. Ogievetsky and E.S. Sokatchev, Harmonic superspace, Cambridge University Press, Cambridge U.K. (2001).

[4] S.J. Gates, M.T. Grisaru, M. Roćek, W. Siegel, Superspace or one Thousand and One Lessons in Supersymmetry, Benjamin Cummings, Reading U.S.A. (1983).

[5] I.L. Buchbinder and S.M. Kuzenko, Ideas and Methods of Supersymmetry and Superfravity or a Walk Through Superspace, IOP Publishing, Bristol U.K. (1998).

[6] M. Dress, R.M. Godbole and P. Roy, Sparticles. An account of four-dimensional $\mathcal{N}=1$ supersymmetry in High Energy Physics, World Scientific, New York U.S.A. (2004).

[7] P. Binetruy, Supersymmetry: Theory, Experiment, Cosmology, Oxford University Press, Oxford U.K. (2006).

[8] J. Terning, Modern Supersymmetry, Clarendon Press, Oxford U.K. (2006).

[9] M. Dine, Supersymmetry and String Theory: Beyond the Standrad Model, Cambridge University Press, Cambridge U.K. (2007).

[10] M.A. Vasiliev, Higher spin gauge theories in various dimensions, Fortsch. Phys. 52 (2004) 702 [hep-th/0401177] [INSPIRE].

[11] D. Sorokin, Introduction to the classical theory of higher spins, AIP Conf. Proc. 767 (2005) 172 [hep-th/0405069] [INSPIRE].

[12] A. Fotopoulos and M. Tsulaia, Gauge Invariant Lagrangians for Free and Interacting Higher Spin Fields. A Review of the BRST formulation, Int. J. Mod. Phys. A 24 (2009) 1 [arXiv:0805.1346] [INSPIRE].

[13] X. Bekaert, S. Cnockaert, C. Iazeolla and M.A. Vasiliev, Nonlinear higher spin theories in various dimensions, hep-th/0503128 [INSPIRE].

[14] X. Bekaert, N. Boulanger and P. Sundell, How higher-spin gravity surpasses the spin two barrier: no-go theorems versus yes-go examples, Rev. Mod. Phys. 84 (2012) 987 [arXiv: 1007.0435] [INSPIRE].

[15] A. Sagnotti, Notes on Strings and Higher Spins, J. Phys. A 46 (2013) 214006 [arXiv:1112.4285] [INSPIRE].

[16] V.E. Didenko and E.D. Skvortsov, Elements of Vasiliev theory, arXiv:1401.2975 [INSPIRE].

[17] M.A. Vasiliev, Higher-Spin Theory and Space-Time Metamorphoses, Lect. Notes Phys. 892 (2015) 227 [arXiv:1404.1948] [INSPIRE].

[18] T. Curtright, Massless Field Supermultiplets With Arbitrary Spin, Phys. Lett. B 85 (1979) 219 [INSPIRE].

[19] S.M. Kuzenko, A.G. Sibiryakov and V.V. Postnikov, Massless gauge superfields of higher half integer superspins, JETP Lett. 57 (1993) 534 [INSPIRE].

[20] S.M. Kuzenko and A.G. Sibiryakov, Massless gauge superfields of higher integer superspins, JETP Lett. 57 (1993) 539 [inSPIRE]. 
[21] S.M. Kuzenko and A.G. Sibiryakov, Free massless higher superspin superfields on the anti-de Sitter superspace, Phys. Atom. Nucl. 57 (1994) 1257 [arXiv:1112.4612] [INSPIRE].

[22] S.J. Gates Jr., S.M. Kuzenko and A.G. Sibiryakov, $\mathcal{N}=2$ supersymmetry of higher superspin massless theories, Phys. Lett. B 412 (1997) 59 [hep-th/9609141] [INSPIRE].

[23] S.J. Gates Jr., S.M. Kuzenko and A.G. Sibiryakov, Towards a unified theory of massless superfields of all superspins, Phys. Lett. B 394 (1997) 343 [hep-th/9611193] [INSPIRE].

[24] S.J. Gates Jr. and K. Koutrolikos, On $4 D, \mathcal{N}=1$ massless gauge superfields of arbitrary superhelicity, JHEP 06 (2014) 098 [arXiv:1310.7385] [INSPIRE].

[25] Yu. M. Zinoviev, Massive $\mathcal{N}=1$ supermultiplets with arbitrary superspins, Nucl. Phys. B 785 (2007) 98 [arXiv:0704.1535] [inSPIRE].

[26] I.L. Buchbinder, T.V. Snegirev and Yu. M. Zinoviev, Lagrangian formulation of the massive higher spin supermultiplets in three dimensional space-time, JHEP 10 (2015) 148 [arXiv: 1508.02829] [INSPIRE].

[27] I.L. Buchbinder, S.J. Gates Jr., W.D. Linch, III and J. Phillips, New $4 D, \mathcal{N}=1$ superfield theory: Model of free massive superspin-3/2 multiplet, Phys. Lett. B 535 (2002) 280 [hep-th/0201096] [INSPIRE].

[28] I.L. Buchbinder, S.J. Gates Jr., W.D. Linch, III and J. Phillips, Dynamical superfield theory of free massive superspin-1 multiplet, Phys. Lett. B 549 (2002) 229 [hep-th/0207243] [INSPIRE].

[29] I.L. Buchbinder, S.J. Gates Jr., S.M. Kuzenko and J. Phillips, Massive $4 D, \mathcal{N}=1$ superspin 1 and 3/2 multiplets and dualities, JHEP 02 (2005) 056 [hep-th/0501199] [INSPIRE].

[30] S.J. Gates Jr. and K. Koutrolikos, A dynamical theory for linearized massive superspin 3/2, JHEP 03 (2014) 030 [arXiv: 1310.7387] [INSPIRE].

[31] E.S. Fradkin and G.A. Vilkovisky, Quantization of relativistic systems with constraints, Phys. Lett. B 55 (1975) 224 [INSPIRE].

[32] I.A. Batalin and G.A. Vilkovisky, Relativistic S Matrix of Dynamical Systems with Boson and Fermion Constraints, Phys. Lett. B 69 (1977) 309 [INSPIRE].

[33] E.S. Fradkin and T.E. Fradkina, Quantization of Relativistic Systems with Boson and Fermion First and Second Class Constraints, Phys. Lett. B 72 (1978) 343 [inSPIRE].

[34] I.A. Batalin and E.S. Fradkin, Operator Quantization of Relativistic Dynamical Systems Subject to First Class Constraints, Phys. Lett. B 128 (1983) 303 [InSPIRE].

[35] M. Henneaux, Hamiltonian Form of the Path Integral for Theories with a Gauge Freedom, Phys. Rept. 126 (1985) 1 [INSPIRE].

[36] I.A. Batalin and E.S. Fradkin, Operatorial quantizaion of dynamical systems subject to constraints. A Further study of the construction, Annales Poincare Phys. Theor. 49 (1988) 145.

[37] I.L. Buchbinder, A. Pashnev and M. Tsulaia, Lagrangian formulation of the massless higher integer spin fields in the AdS background, Phys. Lett. B 523 (2001) 338 [hep-th/0109067] [INSPIRE].

[38] X. Bekaert, I.L. Buchbinder, A. Pashnev and M. Tsulaia, On higher spin theory: Strings, BRST, dimensional reductions, Class. Quant. Grav. 21 (2004) S1457 [hep-th/0312252] [INSPIRE]. 
[39] I.L. Buchbinder, A. Fotopoulos, A.C. Petkou and M. Tsulaia, Constructing the cubic interaction vertex of higher spin gauge fields, Phys. Rev. D 74 (2006) 105018 [hep-th/0609082] [INSPIRE].

[40] A. Fotopoulos, N. Irges, A.C. Petkou and M. Tsulaia, Higher-Spin Gauge Fields Interacting with Scalars: The Lagrangian Cubic Vertex, JHEP 10 (2007) 021 [arXiv:0708.1399] [INSPIRE].

[41] I.L. Buchbinder, P. Dempster and M. Tsulaia, Massive Higher Spin Fields Coupled to a Scalar: Aspects of Interaction and Causality, Nucl. Phys. B 877 (2013) 260 [arXiv: 1308.5539] [INSPIRE].

[42] I.L. Buchbinder and V.A. Krykhtin, Gauge invariant Lagrangian construction for massive bosonic higher spin fields in D dimensions, Nucl. Phys. B 727 (2005) 537 [hep-th/0505092] [INSPIRE].

[43] I.L. Buchbinder, V.A. Krykhtin, L.L. Ryskina and H. Takata, Gauge invariant Lagrangian construction for massive higher spin fermionic fields, Phys. Lett. B 641 (2006) 386 [hep-th/0603212] [INSPIRE].

[44] I.L. Buchbinder, V.A. Krykhtin and P.M. Lavrov, Gauge invariant Lagrangian formulation of higher spin massive bosonic field theory in AdS space, Nucl. Phys. B 762 (2007) 344 [hep-th/0608005] [INSPIRE].

[45] I.L. Buchbinder, V.A. Krykhtin and A. Pashnev, BRST approach to Lagrangian construction for fermionic massless higher spin fields, Nucl. Phys. B 711 (2005) 367 [hep-th/0410215] [INSPIRE].

[46] I.L. Buchbinder, A.V. Galajinsky and V.A. Krykhtin, Quartet unconstrained formulation for massless higher spin fields, Nucl. Phys. B 779 (2007) 155 [hep-th/0702161] [INSPIRE].

[47] I.L. Buchbinder, V.A. Krykhtin and A.A. Reshetnyak, BRST approach to Lagrangian construction for fermionic higher spin fields in (A)dS space, Nucl. Phys. B 787 (2007) 211 [hep-th/0703049] [INSPIRE].

[48] I.L. Buchbinder, V.A. Krykhtin and H. Takata, Gauge invariant Lagrangian construction for massive bosonic mixed symmetry higher spin fields, Phys. Lett. B 656 (2007) 253 [arXiv: 0707.2181] [INSPIRE].

[49] I.L. Buchbinder, V.A. Krykhtin and M. Tsulaia, Lagrangian formulation of massive fermionic higher spin fields on a constant electromagnetic background, Nucl. Phys. B 896 (2015) 1 [arXiv:1501.03278] [InSPIRE].

[50] I.L. Buchbinder and V.A. Krykhtin, Quartic interaction vertex in the massive integer higher spin field theory in a constant electromagnetic field, Eur. Phys. J. C 75 (2015) 454 [arXiv: 1507.03723] [INSPIRE].

[51] O.A. Gelfond and M.A. Vasiliev, $\operatorname{Sp}(8)$ invariant higher spin theory, twistors and geometric BRST formulation of unfolded field equations, JHEP 12 (2009) 021 [arXiv:0901.2176] [INSPIRE].

[52] O.A. Gelfond and M.A. Vasiliev, Unfolding Versus BRST and Currents in $\operatorname{Sp}(2 M)$ Invariant Higher-Spin Theory, arXiv:1001.2585 [INSPIRE].

[53] K.B. Alkalaev and M. Grigoriev, Unified BRST description of AdS gauge fields, Nucl. Phys. B 835 (2010) 197 [arXiv:0910.2690] [INSPIRE]. 
[54] M. Grigoriev and A. Waldron, Massive Higher Spins from BRST and Tractors, Nucl. Phys. B 853 (2011) 291 [arXiv: 1104.4994] [INSPIRE].

[55] K. Alkalaev and M. Grigoriev, Unified BRST approach to (partially) massless and massive AdS fields of arbitrary symmetry type, Nucl. Phys. B 853 (2011) 663 [arXiv:1105.6111] [INSPIRE].

[56] R.R. Metsaev, BRST-BV approach to cubic interaction vertices for massive and massless higher-spin fields, Phys. Lett. B 720 (2013) 237 [arXiv:1205.3131] [INSPIRE].

[57] R.R. Metsaev, BRST invariant effective action of shadow fields, conformal fields and AdS/CFT, Theor. Math. Phys. 181 (2014) 1548 [arXiv:1407.2601] [INSPIRE].

[58] R.R. Metsaev, BRST-BV approach to massless fields adapted to AdS/CFT correspondence, arXiv: 1508.07928 [INSPIRE].

[59] I.A. Batalin and I.V. Tyutin, Existence theorem for the effective gauge algebra in the generalized canonical formalism with Abelian conversion of second class constraints, Int. J. Mod. Phys. A 6 (1991) 3255 [inSPIRE].

[60] E.S. Egorian and R.P. Manvelyan, Quantization of dynamical systems with first and second class constraints, Theor. Math. Phys. 94 (1993) 173 [INSPIRE]. 